CTP-TAMU-09/02

UU-02-02

hep-th/0205132

October 27, 2018

\title{
Analysis of Higher Spin Field Equations in Four Dimensions
}

\author{
E. Sezgin \\ Center for Theoretical Physics, Texas A\&M University, College Station, TX 77843, USA \\ P. Sundell \\ Department for Theoretical Physics, Uppsala Universitet, Sweden
}

\begin{abstract}
The minimal bosonic higher spin gauge theory in four dimensions contains massless particles of spin $s=0,2,4, .$. that arise in the symmetric product of two spin 0 singletons. It is based on an infinite dimensional extension of the $A d S_{4}$ algebra a la Vasiliev. We derive an expansion scheme in which the gravitational gauge fields are treated exactly and the gravitational curvatures and the higher spin gauge fields as weak perturbations. We also give the details of an explicit iteration procedure for obtaining the field equations to arbitrary order in curvatures. In particular, we highlight the structure of all the quadratic terms in the field equations.
\end{abstract}




\section{Introduction}

The correspondence between weakly coupled gauged supergravity in $A d S_{5}$ and strongly coupled supersymmetric Yang-Mills theory living on the four dimensional boundary of $A d S_{5}$ has been intensely studied in recent years and these studies have led to many interesting results. Much less in known, however, in the limit in which the Yang-Mills theory is weakly coupled or even free. Nonetheless, it is well known that in such a limit two things happen. Firstly, the bulk supergravity is no longer a good approximation due to the fact that the mass of the string states become light. Secondly, the free Yang-Mills theory, by which we mean an SU(N) Yang-Mills theory with $g_{Y M} \rightarrow 0$, admits infinitely many conserved currents with ever increasing spin.

The above facts, together with considerations detailed elsewhere, have led to the proposal that free SYM on the boundary of $A d S_{5}$ is holographic dual of a bulk theory which is an interacting higher spin (HS) gauge theory $[1,2,3,4,5,6,7,8,9,10]$ based on an infinite dimensional extension of the AdS superalgebra. It has been furthermore noted that turning on the Yang-Mills coupling constant gives rise to a Higgs mechanism in the bulk in which the HS symmetries are spontaneously broken down to those of the ordinary supergravity, namely the finite dimensional AdS supersymmetry [7, 10]. An interesting twist to this story has appeared recently in [11] where a remarkable connection between higher spins and the dynamics of long, folded closed strings in $A d S_{5}$ has been found. These developments begin to merely scratch the surface of very interesting physics in a new phase string/M theory which has hardly been explored so far, and it holds the promise of exciting results.

In this paper we will neither address in detail the free CFT/HS gauge theory correspondence, nor the construction of the interacting, full 5D HS gauge theory; we have discussed these and several related matters recently in [10]. Instead, here we shall examine closely the structure of the interactions in the simplest possible situation, namely in a minimal bosonic $\mathrm{HS}$ gauge theory in 4D where the full field equations including auxiliary fields have been known in a closed form for some time $[12,13]$. However, the ingredients that go into their formulation are not widely familiar. Moreover, it would be useful to extract the couplings of all the physical fields involved explicitly so that traditional field theory computations may become feasible in order to test various aspects of the proposed CFT/HS gauge theory correspondence. With that in mind, here we shall analyze in greater detail than before the HS equations of motion in the case of the minimal bosonic model in $4 \mathrm{D}$. Further work is required, however, to present them explicitly in terms of physical fields which shows the numerical coefficients of the couplings as well as the distribution of derivatives [14].

The HS gauge theory model involves a star product and in many respects is reminiscent of non-commutative gauge field theories. The reader can find relatively detailed 
historical background and references for HS gauge theories, for example, in [3]. These theories have been primarily been developed by Vasiliev $[12,13]$. In $[15,16]$, we have expressed Vasiliev's main result in a way which makes the geometrical nature of the equations somewhat more transparent. In doing so, however, the precise relation with Vasiliev's equations have not been made altogether precise. This will be remedied here and we will exhibit the precise relation in this paper.

The physical fields of the minimal bosonic model are massless fields with spin $s=$ $0,2,4, \ldots$, each occurring once. For reasons explained elsewhere (see [3], for example, for a review), this is precisely the spectrum which arises in the tensor product of two spin 0 singleton representations of the AdS group $S O(3,2)$ [17]. Extensions of the model to the supersymmetric case as well as higher dimensions show that this bosonic spectrum is always contained as a minimal bosonic truncation $[3,4,9,10]$ (which is consistent in $4 \mathrm{D}$ where the interactions are known). Given also the fact that the minimal bosonic model already exhibits many features of the higher supersymmetric models, it provides an ideal ground for studying the basic properties of HS gauge theory.

The notion of curvature expansion has been introduced by Vasiliev who studied the expansion for a certain 4D HS gauge theory in up to second order in [18]. Here we develop further the generally covariant expansion scheme [19], in which the gravitational gauge fields are treated exactly while all curvatures, higher spin gauge fields and the physical scalar field are treated as weak fields. In particular, we describe a scheme for solving the highly complicated constraint equations for the auxiliary fields to any order in the weak fields.

An action, with or without auxiliary fields, which reproduces the full field equations is not known. It should nevertheless be possible to use the weak field expansion scheme to build an action for the physical fields, including the boundary terms which are important for computing bulk-to-boundary amplitudes. It would also be interesting to exhibit the form of the scalar potential. We hope to address these issues elsewhere.

This paper is organized as follows. In Section 2 we define the minimal bosonic model and describe its full field equations including auxiliary fields in an expansion in terms of the scalar field, the generalized Weyl tensors and their derivatives. In Section 3 we show how the minimal bosonic model can be obtained from the $\mathcal{N}=2$ model of Vasiliev [12] by consistent truncation. In Section 4, we elaborate on the Lorentz transformation of the master fields ensuring that the component fields transform in an appropriate manner [13]. In Section 5, we describe a weak field expansion scheme for eliminating the auxiliary fields and obtaining the interactions among the physical fields to any desired order. In Section 6, we take a closer look at the field equations including second order terms, and exhibit their structure in considerable detail. In Section 7 we comment on our results, holography and some open problems. 


\section{The Minimal Bosonic Model and Curvature Ex- pansion}

The minimal bosonic theory in $4 \mathrm{D}$ is based on the higher spin algebra $h s_{2}(1)$ [21], which we shall refer to in this paper as $h s(4)$. This algebra is realized in terms of oscillators obeying the following algebra

$$
y_{\alpha} \star y_{\beta}=y_{\alpha} y_{\beta}+i \epsilon_{\alpha \beta}, \quad y_{\alpha} \star \bar{y}_{\dot{\alpha}}=y_{\alpha} \bar{y}_{\dot{\alpha}}, \quad\left(y_{\alpha}\right)^{\dagger}=\bar{y}_{\dot{\alpha}}
$$

where $y_{\alpha}(\alpha=1,2)$ is a Weyl spinor which is a Grassmann even generator of a Heisenberg algebra. The $\star$ denotes the associative product between oscillators. The products on the right hand sides are Weyl ordered, so that for example $y_{\alpha} y_{\beta}=y_{\beta} y_{\alpha}$. Using the above contraction rules it is straightforward to compute $f \star g$ where $f(y, \bar{y})$ and $g(y, \bar{y})$ are two arbitrary Weyl ordered polynomials of oscillators (see (2.12) below). The resulting $\star$-algebra

is associative.

The algebra $h s(4)$ consists of arbitrary Grassmann even and anti-hermitian polynomials $P(y, \bar{y}, \theta)$ that are sums of monomials of degree $4 \ell+2$ where $\ell=0,1,2, \ldots$, which will be referred to as the level index. The Lie bracket between $P, Q \in h s(4)$ is given by $[P, Q]_{\star}$. Thus, denoting by $P^{(\ell)}$ an $\ell$ th level monomial, the commutation relations have the schematic form:

$$
\left[P^{\left(\ell_{1}\right)}, P^{\left(\ell_{2}\right)}\right]_{\star}=\sum_{\left|\ell_{1}-\ell_{2}\right| \leq \ell \leq \ell_{1}+\ell_{2}} P^{(\ell)}
$$

In particular, the zeroth level of $h s(4)$ is the maximal finite subalgebra $S O(3,2) \simeq$ $S p(4)$ whose generators schematically take the form

$$
M_{\alpha \dot{\beta}}=y_{\alpha} \bar{y}_{\dot{\beta}}, \quad M_{\alpha \beta}=y_{\alpha} y_{\beta}, \quad M_{\dot{\alpha} \dot{\beta}}=\bar{y}_{\dot{\alpha}} \bar{y}_{\dot{\beta}}
$$

A generator $P^{(\ell)}$ in the $\ell$ th level of $h s(4)$ can be expanded as

$$
P^{(\ell)}(y, \bar{y}, \theta)=\sum_{\substack{m+n \\=4 \ell+2}} \frac{1}{m ! n !} \bar{y}^{\dot{\alpha}_{1}} \cdots \bar{y}^{\dot{\alpha}_{m}} y^{\beta_{1}} \cdots y^{\beta_{n}} P_{\dot{\alpha}_{1} \ldots \dot{\alpha}_{m} \beta_{1} \ldots \beta_{n}}
$$

The spins of the components are given by $s=\frac{1}{2}(m+n)$. The reality properties follow from $P^{\dagger}=-P$. It is useful to summarize the conditions that define $P$ as 


$$
\tau(P)=-P, \quad P^{\dagger}=-P
$$

where the $\tau$-map is defined as

$$
\tau(y)=i y, \quad \tau(\bar{y})=i \bar{y}
$$

The $\tau$-map obeys $\tau(f \star g)=\tau(g) \star \tau(f)$ which makes it an anti- involution of the associative $\star$-algebra.

The basic building blocks of the HS gauge theory based on this algebra are the master 1-form $A(x ; y, \bar{y})=d x^{\mu} A_{\mu}(x ; y, \bar{y})$, where $x^{\mu}$ are the coordinates of the $4 \mathrm{D}$ spacetime, and a master 0 -form $\Phi(x ; y, \bar{y})$ which satisfy the conditions

$$
\tau\left(A_{\mu}\right)=-A_{\mu}, \quad\left(A_{\mu}\right)^{\dagger}=-A_{\mu}, \quad \tau(\Phi)=\bar{\pi}(\Phi), \quad \Phi^{\dagger}=\pi(\Phi)
$$

where the maps $\pi$ and $\bar{\pi}$ maps are defined as

$$
\begin{array}{ll}
\pi\left(y_{\alpha}\right)=-y_{\alpha}, & \bar{\pi}\left(y_{\alpha}\right)=y_{\alpha}, \\
\pi\left(\bar{y}_{\dot{\alpha}}\right)=\bar{y}_{\dot{\alpha}}, & \bar{\pi}\left(\bar{y}_{\dot{\alpha}}\right)=-\bar{y}_{\dot{\alpha}} .
\end{array}
$$

These maps obey $\pi(f \star g)=\pi(f) \star \pi(g)$ and $\bar{\pi}(f \star g)=\bar{\pi}(f) \star \bar{\pi}(g)$ which make them involutions of the associative $\star$-algebra. The rationale behind (2.8) is explained in detail, for example, in [19]. The conditions on $A_{\mu}$ define the adjoint representation of the higher spin algebra $h s(4)$ while the conditions on $\Phi$ define a quasi-adjoint representation of $h s(4)$. The $\pi$-twist is essential for obtaining the physical spectrum of states. The conditions on $A_{\mu}$ ensure that the gauge fields contained in it have even integer spins $s=2,4,6, \ldots$ define an $h s(4)$-valued spacetime one-form and a spacetime zero-form in a certain quasi-adjoint representation of $h s(4)$ [19]. The component field expansion of the master fields reads ${ }^{1}$ :

$$
\begin{aligned}
A_{\mu}(x ; y, \bar{y}) & =\frac{1}{2 i} \sum_{m+n=2 \bmod 4} \frac{1}{m ! n !} \bar{y}^{\dot{\alpha}_{1}} \cdots \bar{y}^{\dot{\alpha}_{m}} y^{\alpha_{1}} \cdots y^{\alpha_{n}} A_{\mu \alpha_{1} \ldots \alpha_{n} \dot{\alpha}_{1} \ldots \dot{\alpha}_{m}}(x), \\
\Phi(x ; y, \bar{y}) & =\sum_{|m-n|=0 \bmod 4} \frac{1}{m ! n !} \bar{y}^{\dot{\alpha}_{1}} \cdots \bar{y}^{\dot{\alpha}_{m}} y^{\alpha_{1}} \cdots y^{\alpha_{n}} \Phi_{\alpha_{1} \ldots \alpha_{n} \dot{\alpha}_{1} \ldots \dot{\alpha}_{m}}(x) .
\end{aligned}
$$

\footnotetext{
${ }^{1}$ From the condition $\tau(\Phi)=\bar{\pi}(\Phi)$ it follows that $\bar{\pi}(\Phi)=\pi(\Phi)$, so that $\tau(\Phi)=\bar{\pi}(\Phi)$ and $\tau(\Phi)=$ $\pi(\Phi)$ give rise to equivalent representations.
} 
To describe the field equations in 4D spacetime one introduces an auxiliary set of coordinates $Z=\left(z^{\alpha}, \bar{z}^{\dot{\alpha}}\right)$ which are Grassmann even spinors that are non-commutative in nature [12]. To be more precise, in addition to (2.1), one postulates the $\star$-commutation rules

$$
z_{\alpha} \star z_{\beta}=z_{\alpha} z_{\beta}-i \epsilon_{\alpha \beta}, \quad y_{\alpha} \star z_{\beta}=y_{\alpha} z_{\beta}-i \epsilon_{\alpha \beta}, \quad z_{\alpha} \star y_{\beta}=z_{\alpha} y_{\beta}+i \epsilon_{\alpha \beta}
$$

and their complex conjugates with $\left(z_{\alpha}\right)^{\dagger}=\bar{z}_{\dot{\alpha}}$. The resulting associative $\star$-product between two arbitrary Weyl-ordered polynomials $\widehat{f}(y, \bar{y} ; z, \bar{z})$ and $\widehat{g}(y, \bar{y} ; z, \bar{z})$ is defined as

$$
\widehat{f} * \widehat{g}=\widehat{f} \exp \left[i\left(\frac{\overleftarrow{\partial}}{\partial z_{\alpha}}+\frac{\overleftarrow{\partial}}{\partial y_{\alpha}}\right)\left(\frac{\vec{\partial}}{\partial z^{\alpha}}-\frac{\vec{\partial}}{\partial y^{\alpha}}\right)+i\left(\frac{\overleftarrow{\partial}}{\partial \bar{z}_{\dot{\alpha}}}-\frac{\overleftarrow{\partial}}{\partial \bar{y}_{\dot{\alpha}}}\right)\left(\overrightarrow{\frac{\partial}{\partial \bar{z}^{\dot{\alpha}}}}+\frac{\vec{\partial}}{\partial \bar{y}^{\dot{\alpha}}}\right)\right] \widehat{g}
$$

Here and in the rest of the paper, hatted quantities depend on $(z, \bar{z})$. One then considers extensions $\widehat{A}$ and $\widehat{\Phi}$ of the basic master fields to the $(x ; z, \bar{z})$ space such that $A=$ $\left.\widehat{A}\right|_{Z=0}$ and $\Phi=\left.\widehat{\Phi}\right|_{Z=0}$. One then imposes constraints on curvatures $\widehat{F}$ and $\widehat{D} \widehat{\Phi}$ (see (2.17), (2.18), (2.19) and (2.20) below) whose $(z, \bar{z})$-components determine the $(z, \bar{z})$ dependence of $\widehat{A}$ and $\widehat{\Phi}$ in terms of $A$ and $\Phi$. The remaining, non-trivial curvature constraints are contained in the reduced curvature constraints $\left.\widehat{F}\right|_{Z=0}$ and $\left.\widehat{D} \widehat{\Phi}\right|_{Z=0}$ (see (2.40) and (2.41) below), which are integrable by construction and one can show that they contain the physical field equations of the HS gauge theory. It is important to note that since $(z, \bar{z})$ are non-commutative the reduced constraints contain highly nontrivial interactions even though the original constraint in $(x ; z, \bar{z})$ space has a simple form.

The extensions of the basic master fields are a 0-form $\widehat{\Phi}$ and a 1-form

$$
\widehat{A}=d x^{\mu} \widehat{A}_{\mu}+d z^{\alpha} \widehat{A}_{\alpha}+d \bar{z}^{\dot{\alpha}} \widehat{A}_{\dot{\alpha}}
$$

The component fields of $\widehat{A}$ and $\widehat{\Phi}$ are functions of $(x ; z, \bar{z})$ with $(y, \bar{y})$ expansions obeying the conditions

$$
\tau(\widehat{A})=-\widehat{A}, \quad \widehat{A}^{\dagger}=-\widehat{A}, \quad \tau(\widehat{\Phi})=\bar{\pi}(\widehat{\Phi}), \quad \widehat{\Phi}^{\dagger}=\pi(\widehat{\Phi})
$$

where the anti-involution $\tau$ and involution $\pi$ and $\bar{\pi}$ are defined by (2.6), (2.8), and 


$$
\begin{array}{llll}
\pi\left(z_{\alpha}\right)=-z_{\alpha}, & \bar{\pi}\left(z_{\alpha}\right)=z_{\alpha}, & \tau\left(z_{\alpha}\right)=-i z_{\alpha}, \\
\pi\left(\bar{z}_{\dot{\alpha}}\right)=\bar{z}_{\dot{\alpha}}, & \bar{\pi}\left(\bar{z}_{\dot{\alpha}}\right)=-\bar{z}_{\dot{\alpha}}, & \tau\left(\bar{z}_{\dot{\alpha}}\right)=-i \bar{z}_{\dot{\alpha}},
\end{array}
$$

and that $d(\tau(\widehat{f}))=\tau(d(\widehat{f}))$, idem $\pi$ and $\bar{\pi}$. Thus, the hatted fields are given as expansions order by order in $z$ and $\bar{z}$, with expansion coefficients that in turn are functions of $x$ with $(y, \bar{y})$ expansions obeying (2.14). These conditions are engineered such that at $Z \equiv(z, \bar{z})=0$, the pulled-back components

$$
A_{\mu}=\left.\widehat{A}_{\mu}\right|_{Z=0}, \quad \Phi=\left.\widehat{\Phi}\right|_{Z=0}
$$

can be identified with the basic master defined in (2.7).

The general method for formulating master curvature constraints giving rise to full higher spin field equations in $D=4$ has been developed by Vasiliev [12]. In particular, a non-minimal bosonic model whose spectrum contains massless physical fields with spin $s=0,1,2, \ldots$, each occurring once, has been described in [20]. Here we eliminate the odd spins by imposing conditions given in (2.7), thereby obtaining the minimal bosonic model. These conditions are formally the same as those imposed on the basic master fields of the minimal $\mathcal{N}=8$ model, which was studied in great detail in $[15,16]$. Naturally, the master curvature constraints of the minimal bosonic model also take the same form as those of the $\mathcal{N}=8$ model. Of course, the fermionic oscillators of the $\mathcal{N}=8$ model are to be dropped and the $S O(8)$ chirality operator $\Gamma$ occurring in that model is to be replaced by unity. Thus the master curvature constraints giving rise to the full field equations for the minimal bosonic theory are

$$
\begin{aligned}
\widehat{F} & =\frac{i}{4} d z^{\alpha} \wedge d z_{\alpha} \mathcal{V}(\widehat{\Phi} \star \kappa)+\frac{i}{4} d \bar{z}^{\dot{\alpha}} \wedge d \bar{z}_{\dot{\alpha}} \overline{\mathcal{V}}(\widehat{\Phi} \star \bar{\kappa}) \\
\widehat{D} \widehat{\Phi} & =0
\end{aligned}
$$

where the curvatures are defined as

$$
\begin{aligned}
\widehat{F} & =d \widehat{A}+\widehat{A} \star \widehat{A} \\
\widehat{D} \widehat{\Phi} & =d \widehat{\Phi}+\widehat{A} \star \widehat{\Phi}-\widehat{\Phi} \star \bar{\pi}(\widehat{A}) .
\end{aligned}
$$

and the operators $\kappa, \bar{\kappa}$ as 


$$
\kappa=\exp \left(i y^{\alpha} z_{\alpha}\right), \quad \bar{\kappa}=\kappa^{\dagger}=\exp \left(-i \bar{y}^{\dot{\alpha}} \bar{z}_{\dot{\alpha}}\right)
$$

The quantity $\mathcal{V}(X)$ in $(2.17)$ is a $\star$-function, $\mathcal{V}(X)=b_{0}+b_{1} X+b_{2} X \star X+\cdots$, with its complex conjugate $\mathcal{V}(X)$ defined by $\overline{\mathcal{V}}(X)=\left(\mathcal{V}\left(X^{\dagger}\right)\right)^{\dagger}$. The requirement that the theory admits proper local Lorentz transformations [13], as we shall discuss in detail in Section 4, reduces the amount of freedom in $\mathcal{V}$. The possibility of redefining $\widehat{\Phi}$ while preserving the basic structure of the curvature constraint $(2.17)[12,16]$ leads to further reductions in the number of free parameters in $\mathcal{V}$. The result is that $\mathcal{V}$ is an odd function,

$$
\mathcal{V}(X)=b_{1} X+b_{3} X \star X \star X+\cdots,
$$

which is defined modulo the field redefinitions $\widehat{\Phi} \rightarrow F(\widehat{\Phi})$ where $F$ is a real and odd $\star$-function, i.e.

$$
\mathcal{V}(X) \sim \mathcal{V}(F(X)), \quad F(X)=-F(-X), \quad(F(X))^{\dagger}=F\left(X^{\dagger}\right)
$$

As a result there is no loss of generality in setting

$$
\left|b_{1}\right|=1, \quad b_{1}=e^{i \theta_{1}}
$$

We shall see that even the simplest choice $\mathcal{V}(X)=b_{1} X$ leads to highly complicated interactions which are higher order in derivatives. Adding higher order terms to $\mathcal{V}(X)$ lead to modifications of the higher order interactions, which are, of course, consistent with HS gauge invariance. The free parameters in $\mathcal{V}$ are therefore genuine interaction ambiguities. It has been proposed that these parameters can be fixed upon comparison with a underlying singleton theory [10].

It is important to note that the constraints (2.17) and (2.18) are integrable and have the symmetry

$$
\begin{aligned}
\delta \widehat{A} & =d \widehat{\epsilon}+[\widehat{A}, \widehat{\epsilon}]_{\star}, \\
\delta \widehat{\Phi} & =\widehat{\Phi} \star \bar{\pi}(\widehat{\epsilon})-\widehat{\epsilon} \star \widehat{\Phi},
\end{aligned}
$$

where $\widehat{\epsilon}$ obeys the same conditions as those imposed on $\widehat{A}$ in (2.14), that is

$$
\tau(\widehat{\epsilon})=-\widehat{\epsilon}, \quad(\widehat{\epsilon})^{\dagger}=-\widehat{\epsilon}
$$


The components of the constraints (2.17) and (2.18) which have at least one $z^{\alpha}$ or $\bar{z}^{\dot{\alpha}}$ index determine the $Z$-dependence of $\widehat{A}$ and $\widehat{\Phi}$ in terms of the initial conditions $(2.16)$ up to a gauge transformation. By assuming that $\widehat{\Phi}$ and $\widehat{A}$ can be expanded in powers of $\Phi$, which contains curvatures and the scalar field, we can obtain the $Z$-dependence of the master fields by making the following ansatz:

$$
\widehat{\Phi}=\sum_{n=1}^{\infty} \widehat{\Phi}^{(n)}, \quad \widehat{A}_{\alpha}=\sum_{n=0}^{\infty} \widehat{A}_{\alpha}^{(n)}, \quad \widehat{A}_{\mu}=\sum_{n=0}^{\infty} \widehat{A}_{\mu}^{(n)}
$$

where $\widehat{\Phi}^{(n)}(n=1,2,3, \ldots), \widehat{A}_{\alpha}^{(n)}(n=0,1,2, \ldots)$ and $\widehat{A}_{\mu}^{(n)}(n=0,1,2, \ldots)$ are funcionals which are $n$th order in $\Phi$ and which obey the initial conditions

$$
\begin{aligned}
& \left.\widehat{\Phi}^{(n)}\right|_{Z=0}= \begin{cases}\Phi, & n=1 \\
0, & n=2,3, \ldots\end{cases} \\
& \left.\widehat{A}_{\mu}^{(n)}\right|_{Z=0}= \begin{cases}A_{\mu}, & n=0 \\
0, & n=1,2,3, \ldots\end{cases}
\end{aligned}
$$

¿From $\widehat{F}_{\alpha \beta}^{(0)}=0=\widehat{F}_{\alpha \dot{\beta}}^{(0)}$ it follows that $\widehat{A}_{\alpha}^{(0)}$ is a pure gauge artifact which we can eliminate by imposing the gauge condition

$$
\widehat{A}_{\alpha}^{(0)}=0
$$

Next, the constraints $\widehat{F}_{\alpha \dot{\beta}}=0, \widehat{F}_{\alpha \beta}=-\frac{i}{2} \epsilon_{\alpha \beta} \mathcal{V}(\widehat{\Phi} \star \kappa)$ and $\widehat{D}_{\alpha} \widehat{\Phi}=0$ can be solved in the $n$th order $(n \geq 1)$ as

$$
\begin{aligned}
\widehat{\Phi}^{(1)} & =\Phi(y, \bar{y}) \\
\widehat{\mathcal{A}}_{\alpha}^{(1)} & =\partial_{\alpha} \widehat{\xi}^{(1)}-\frac{i b_{1}}{2} z_{\alpha} \int_{0}^{1} t d t \Phi(-t z, \bar{y}) \kappa(t z, y)
\end{aligned}
$$

and $(n \geq 2)$ :

$$
\begin{aligned}
\widehat{\Phi}^{(n)}= & z^{\alpha} \sum_{j=1}^{n-1} \int_{0}^{1} d t\left(\widehat{\Phi}^{(j)} \star \bar{\pi}\left(\widehat{A}_{\alpha}^{(n-j)}\right)-\widehat{A}_{\alpha}^{(n-j)} \star \widehat{\Phi}^{(j)}\right)_{z \rightarrow t z, \bar{z} \rightarrow t \bar{z}} \\
& +\bar{z}^{\dot{\alpha}} \sum_{j=1}^{n-1} \int_{0}^{1} d t\left(\widehat{\Phi}^{(j)} \star \pi\left(\widehat{A}_{\dot{\alpha}}^{(n-j)}\right)-\widehat{A}_{\dot{\alpha}}^{(n-j)} \star \widehat{\Phi}^{(j)}\right)_{z \rightarrow t z, \bar{z} \rightarrow t \bar{z}},
\end{aligned}
$$




$$
\begin{aligned}
\widehat{A}_{\alpha}^{(n)}= & \partial_{\alpha} \widehat{\xi}^{(n)}+z_{\alpha} \int_{0}^{1} t d t\left(-\frac{i}{2} \mathcal{V}^{(n)}(\widehat{\Phi} \star \kappa)+\sum_{j=1}^{n-1} \widehat{A}^{(j) \beta} \star \widehat{A}_{\beta}^{(n-j)}\right)_{z \rightarrow t z, \bar{z} \rightarrow t \bar{z}} \\
& +\bar{z}^{\dot{\beta}} \sum_{j=1}^{n-1} \int_{0}^{1} t d t\left[\widehat{A}_{\alpha}^{(j)}, \widehat{A}_{\dot{\beta}}^{(n-j)}\right]_{*(z \rightarrow t z, \bar{z} \rightarrow t \bar{z})},
\end{aligned}
$$

where $\mathcal{V}^{(n)}(\widehat{\Phi} \star \kappa)$ is the $n$th order term of $\mathcal{V}(\widehat{\Phi} \star \kappa)$ in the curvature expansion scheme (2.28). We emphasize that in (2.38), (2.34) and (2.35) the replacements $(z, \bar{z})$ by $(t z, t \bar{z})$ are to be made after the $\star$-products are carried out. The integration functions $\widehat{\xi}^{(n)}$ are gauge artifacts which can be eliminated by means of $\Phi$-dependent gauge transformations. We therefore impose the gauge conditions [15]

$$
\widehat{\xi}^{(n)}=0, \quad n=1,2, \ldots
$$

The gauge conditions (2.31) and (2.36) are left invariant by $Z$-independent, and therefore $h s(4)$-valued, gauge transformations (which in general may be $\Phi$-dependent).

¿From the constraint $\widehat{F}_{\mu \alpha}=0$ in (2.17) it follows that

$$
\widehat{A}_{\mu}=\frac{1}{1+\widehat{L}^{(1)}+\widehat{L}^{(2)}+\widehat{L}^{(3)}+\cdots} A_{\mu},
$$

where the linear operators $\widehat{L}^{(n)}$ are defined by

$$
\begin{aligned}
\widehat{L}^{(n)}(\widehat{f})=-i \int_{0}^{1} \frac{d t}{t} & {\left[\widehat{A}^{\alpha(n)} \star\left(\frac{\partial \widehat{f}}{\partial z^{\alpha}}-\frac{\partial \widehat{f}}{\partial y^{\alpha}}\right)+\left(\frac{\partial \widehat{f}}{\partial z^{\alpha}}+\frac{\partial \widehat{f}}{\partial y^{\alpha}}\right) \star \widehat{A}^{\alpha(n)}\right.} \\
& \left.+\widehat{A}^{\dot{\alpha}(n)} \star\left(\frac{\partial \widehat{f}}{\partial \bar{z}^{\dot{\alpha}}}+\frac{\partial \widehat{f}}{\partial \bar{y}^{\dot{\alpha}}}\right)+\left(\frac{\partial \widehat{f}}{\partial \bar{z}^{\dot{\alpha}}}-\frac{\partial \widehat{f}}{\partial \bar{y}^{\dot{\alpha}}}\right) \star \widehat{A}^{\dot{\alpha}(n)}\right]_{z \rightarrow t z, \bar{z} \rightarrow t \bar{z}}
\end{aligned}
$$

Note that diffeomorphism invariance in $x^{\mu}$ requires $\widehat{A}_{\mu}$ to be linear in $A_{\mu}$ or $\partial_{\mu} \Phi$. However, the gauge conditions (2.31) and (2.36) imply that the $\partial_{\mu} \Phi$ terms cancel identically [19].

Finally, having solved the $Z$-space part of (2.17) and (2.18), the remaining constraints $\widehat{F}_{\mu \nu}=0$ and $\widehat{D}_{\mu} \widehat{\Phi}=0$ yield the following spacetime field equations ${ }^{2}$ :

\footnotetext{
${ }^{2}$ The integrability of (2.17) and (2.18) implies that if $\left.\widehat{F}_{\mu \nu}\right|_{Z=0}=0$ and $\left.\widehat{D}_{\mu} \widehat{\Phi}\right|_{Z=0}=0$ then $\widehat{F}_{\mu \nu}=0$ and $\widehat{D}_{\mu} \widehat{\Phi}=0$.
} 


$$
\begin{aligned}
F_{\mu \nu} & =-2 \sum_{n=1}^{\infty} \sum_{j=0}^{n}\left(\widehat{A}_{[\mu}^{(j)} \star \widehat{A}_{\nu]}^{(n-j)}\right)_{\left.\right|_{Z=0}}, \\
D_{\mu} \Phi & =\sum_{n=2}^{\infty} \sum_{j=1}^{n}\left(\widehat{\Phi}^{(j)} \star \bar{\pi}\left(\widehat{A}_{\mu}^{(n-j)}\right)-\widehat{A}_{\mu}^{(n-j)} \star \widehat{\Phi}^{(j)}\right)_{\left.\right|_{Z=0}}
\end{aligned}
$$

where

$$
F=d A+A \star A, \quad D \Phi=d \Phi+A \star \Phi-\Phi \star \bar{\pi}(A) .
$$

It is important that (2.40) and (2.41) are integrable equations. As such they are invariant under gauge transformations whose form follow uniquely by functional variation of (2.40) and (2.41), as is discussed in more detail at the end of Section 5. Equivalently, these symmetries can be described as the residual $h s(4)$-valued gauge transformations discussed above.

Before we proceed further to analyze in more detail the structure of these equations, it is useful to compare the constraints (2.17) and (2.18) with those of Vasiliev [12].

\section{The Minimal Bosonic Theory from Vasiliev's $\mathcal{N}=$ 2 Model}

The original formulation of the HS field equations in $D=4[12]$ is based on the $\mathcal{N}=2$ higher spin algebra $h u(1,1 \mid 4)[21]$, which is obtained by adding two extra elements $k$ and $\bar{k}$, which are known as Kleinian operators, with the following properties ${ }^{3}$

$$
k^{2}=\bar{k}^{2}=1, \quad[k, \bar{k}]=\left[k, \bar{y}_{\dot{\alpha}}\right]=\left\{k, y_{\alpha}\right\}=\left[\bar{k}, y_{\alpha}\right]=\left\{\bar{k}, \bar{y}_{\dot{\alpha}}\right\}=0
$$

The corresponding enlarged master fields, which we shall denote here by $B_{\mu}$ and $\Psi$, were taken in [12] to obey the reality conditions

$$
\left(B_{\mu}\right)^{\dagger}=-B_{\mu}, \quad(\Psi)^{\dagger}=\Psi
$$

The maximal finite subalgebra of $h u(1,1 \mid 4)$ is $O S p(2 \mid 4) \times U(1)$ with supercharges and $U(1)_{R}$ generator given by [13]:

\footnotetext{
${ }^{3}$ More recently, $\mathcal{N} \geq 1$ models formulated without Kleinian operators have been discussed in [23].
} 


$$
Q_{\alpha}^{1}=y_{\alpha}, \quad Q_{\alpha}^{2}=k \bar{k} y_{\alpha}, \quad J=k \bar{k}
$$

and the extra $U(1)$ generator given by 1 .

The model is then extended into the $Z$-space described in the previous section, with analogs of (3.1) holding also between the Kleinians and $\left(z_{\alpha}, \bar{z}_{\dot{\alpha}}\right)$. The basic master constraints first given in [12] are equivalent to the curvature constraint

$$
\begin{aligned}
d \widehat{B}+\widehat{B} \star \widehat{B} & =\frac{i}{4} d z^{2} \mathcal{V}(\widehat{\Psi}) \star(k \kappa)+\frac{i}{4} d \bar{z}^{2} \overline{\mathcal{V}}(\widehat{\Psi}) \star(\bar{k} \bar{\kappa}) \\
d \widehat{\Psi}+[\widehat{B}, \widehat{\Psi}]_{\star} & =0
\end{aligned}
$$

where $\widehat{B}=d x^{\mu} \widehat{B}_{\mu}+d z^{\alpha} \widehat{B}_{\alpha}+d \bar{z}^{\dot{\alpha}} \widehat{B}_{\dot{\alpha}}$ and $\widehat{\Psi}$ are extensions of $B$ and $\Psi$ obeying

$$
(\widehat{B})^{\dagger}=-\widehat{B}, \quad(\widehat{\Psi})^{\dagger}=\widehat{\Psi}
$$

The dependence of the master fields on the Kleinians results in extra auxiliary as well as physical fields, compared with the minimal bosonic model. Letting

$$
\begin{aligned}
\widehat{B}_{\mu}(k, \bar{k}) & =\widehat{B}_{0 \mu}(k, \bar{k})+\widehat{B}_{1 \mu}(k, \bar{k}), \quad \widehat{B}_{r \mu}(-k,-\bar{k})=(-1)^{r} \widehat{B}_{r \mu}(k, \bar{k}), \\
\widehat{\Psi}(k, \bar{k}) & =\widehat{\Psi}_{0}(k, \bar{k})+\widehat{\Psi}_{1}(k, \bar{k}), \quad \widehat{\Psi}_{r}(-k,-\bar{k})=(-1)^{r} \widehat{\Psi}_{r}(k, \bar{k}),
\end{aligned}
$$

one can show that the initial conditions $B_{1 \mu}$ and $\Psi_{0}$ contain no physical fields [12], i.e. all components of these fields are auxiliary at the linearized level. Moreover, from the structure of (3.4) and (3.5) it follows that

$$
\widehat{B}_{1 \mu}=0, \quad \widehat{\Psi}_{0}=0
$$

is a consistent truncation provided that $\mathcal{V}(X)$ obeys (2.22). The remaining master fields $B_{0 \mu}$ and $\Psi_{1}$ contain physical fields which fill up $O S p(2 \mid 4)$ supermultiplets.

This model can be truncated further to the minimal bosonic model by first imposing

$$
\tau\left(B_{0 \mu}\right)=-B_{0 \mu}, \quad \tau(\Psi)=\Psi
$$

where $\tau(k)=k$ and $\tau(\bar{k})=\bar{k}$, and then identifying

$$
k \bar{k} \simeq 1
$$


¿From (3.10) it follows that

$$
B_{0 \mu}=A_{\mu}^{\prime}+k \bar{k} A_{\mu}^{\prime \prime}, \quad \Psi_{1}=\chi k+\bar{\pi}\left(\chi^{\dagger}\right) \bar{k},
$$

where $A_{\mu}^{\prime}$ and $A_{\mu}^{\prime \prime}$ obeys (2.7) and $\chi$ is unconstrained. Note that the generators (3.3) are dropped from the expansion of $A_{\mu}^{\prime}$ and $A_{\mu}^{\prime \prime}$. Using (3.11) we can identify the master fields $A_{\mu}$ and $\Phi$ obeying $(2.7)$ as

$$
B_{0 \mu} \sim A_{\mu}^{\prime}+A_{\mu}^{\prime \prime} \equiv A_{\mu}, \quad \Psi_{1} k \sim \Psi_{1} \bar{k} \sim \chi+\bar{\pi}\left(\chi^{\dagger}\right) \equiv \Phi .
$$

We conclude that the $\mathcal{N}=2$ master constraints (3.4) and (3.5) of the $h u(1,1 \mid 4)$ model can be truncated consistently to those of the minimal bosonic $h s(4)$ model by means of $(3.9),(3.10)$ and $(3.11)$ provided that $\mathcal{V}$ obeys $(2.22)$.

\section{Lorentz Transformation of the Master Fields}

Turning to the minimal 4D bosonic HS gauge theory described in Section 2, an analysis of the constraints (2.40) and (2.41) to derive the full field equations requires, among other things, an identification of component fields that transform properly under Lorentz transformation. The fact that the component fields of the naive $y$ and $\bar{y}$ expansion of $A_{\mu}$ do not transform as conventional Lorentz tensors was first pointed out in [13] where the field redefinition which defines the proper Lorentz tensors in $A_{\mu}$ was first given.

Following [13], we begin by extending the Lorentz generator $M_{\alpha \beta}$ defined in (2.3) to

$$
\widehat{M}_{\alpha \beta}=y_{\alpha} y_{\beta}-z_{\alpha} z_{\beta}
$$

Commutation with $\widehat{M}_{\alpha \beta}$ generates Lorentz rotations on $y_{\alpha}$ and $z_{\alpha}$. The associated Lorentz parameter is

$$
\widehat{\epsilon}_{0}(\Lambda)=\frac{1}{4 i} \Lambda^{\alpha \beta} \widehat{M}_{\alpha \beta}-\text { h.c. . }
$$

Next, it is important to examine how $\widehat{A}_{\alpha}$ transforms under this Lorentz transformation, especially in view of the fact that this master field carries a free spinorial index and that the gauge choice $(2.31)$ is to be maintained. Under $\widehat{\epsilon}_{0}(\Lambda)$ transformations

$$
\delta \widehat{A}_{\alpha}=\left[\widehat{\epsilon}_{0}(\Lambda), \widehat{A}_{\alpha}\right]_{*}+\frac{1}{2 i} \Lambda_{\alpha}^{\beta} z_{\beta}
$$


This shows that the free spinorial index of $\widehat{A}_{\alpha}$ does not rotate properly under $\widehat{\epsilon}_{0}(\Lambda)$ transformations, and that as a result the transformed field no longer satisfy (2.31). Therefore these transformations need to be modified. To do so, it is convenient to define [12]

$$
\widehat{S}_{\alpha}=z_{\alpha}-2 i \widehat{A}_{\alpha}
$$

and rewrite (4.3) as

$$
\delta \widehat{A}_{\alpha}=\left[\widehat{\epsilon}_{0}(\Lambda), \widehat{A}_{\alpha}\right]_{*}+\Lambda_{\alpha}{ }^{\beta} \widehat{A}_{\beta}+\frac{1}{2 i} \Lambda_{\alpha}{ }^{\beta} \widehat{S}_{\beta}
$$

The first two terms on the right hand side have the desired form but the last term needs to be eliminated. This is achieved with the following extra gauge transformation

$$
\widehat{\epsilon}_{\text {extra }}(\Lambda)=\frac{1}{8 i} \Lambda^{\alpha \beta}\left\{\widehat{S}_{\alpha}, \widehat{S}_{\beta}\right\}_{*}-\text { h.c. }
$$

provided that $\mathcal{V}(X)$ in (2.17) obeys (2.22). This requirement, together with the $\alpha$ component of the constraint (2.18) which implies $S_{\alpha} \star \widehat{\Phi} \star \kappa=-\widehat{\Phi} \star \kappa \star \widehat{S}_{\alpha}$, ensures that

$$
\widehat{S}_{\alpha} \star \mathcal{V}(\widehat{\Phi} \star \kappa)+\mathcal{V}(\widehat{\Phi} \star \kappa) \star \widehat{S}_{\alpha}=0
$$

which in turn implies that the quantity $\frac{1}{2}\left\{\widehat{S}_{\alpha}, \widehat{S}_{\beta}\right\}_{\star}$ appearing in (4.10) generates a Lorentz rotation of $\widehat{S}_{\alpha}$ with opposite sign:

$$
\left[\frac{1}{2}\left\{\widehat{S}_{\alpha}, \widehat{S}_{\beta}\right\}_{\star}, \widehat{S}_{\gamma}\right]_{\star}=-4 i \widehat{S}_{(\alpha} \epsilon_{\beta) \gamma}
$$

It follows that (4.5) removes the unwanted last term in (4.5) ${ }^{4}$ :

$$
\delta_{\text {extra }} \widehat{A}_{\alpha}=-\frac{1}{2 i}\left[\widehat{S}_{\alpha}, \widehat{\epsilon}_{\text {extra }}(\Lambda)\right]_{\star}=-\frac{1}{2 i} \Lambda_{\alpha}{ }^{\beta} \widehat{S}_{\beta}
$$

Thus, we conclude that the appropriate local Lorentz transformation is

\footnotetext{
${ }^{4}$ Note that (2.22) is not needed for the integrability of the constraints (2.17) and (2.18), which only requires $\widehat{D}_{\dot{\alpha}} \mathcal{V}(\widehat{\Phi} \star \kappa)=0$ which in turn implies $\widehat{D}_{\dot{\alpha}} \widehat{\Phi}=0$ for arbitrary $\mathcal{V}(X)$. As for Vasiliev's original $\mathcal{N}=2$ model described in Section 3, the Lorentz invariance of (3.4) and (3.5) requires $\widehat{D}_{\alpha} \mathcal{V}(\widehat{\Psi})=0$ which holds by virtue of $\widehat{D}_{\alpha} \widehat{\Psi}=0$ for arbitrary $\mathcal{V}(X)$.
} 


$$
\begin{aligned}
\widehat{\epsilon}_{L} & =\widehat{\epsilon}_{0}(\Lambda)+\widehat{\epsilon}_{\text {extra }}(\Lambda) \\
& =\frac{1}{4 i} \Lambda^{\alpha \beta}\left(\widehat{M}_{\alpha \beta}+\frac{1}{2}\left\{\widehat{S}_{\alpha}, \widehat{S}_{\beta}\right\}_{\star}\right)-\text { h.c. } \\
& =\frac{1}{4 i} \Lambda^{\alpha \beta}\left(y_{\alpha} y_{\beta}-4 \widehat{A}_{\alpha} \star \widehat{A}_{\beta}\right)-\text { h.c. }
\end{aligned}
$$

Considering the master fields $\widehat{\Phi}$ and $\widehat{A}_{\mu}$ as well, all in all, we get the $\widehat{\epsilon}_{L}(\Lambda)$ transformations

$$
\begin{aligned}
\delta_{L} \widehat{\Phi} & =\left[\widehat{\epsilon}_{0}(\Lambda), \widehat{\Phi}\right]_{\star} \\
\delta_{L} \widehat{A}_{\alpha} & =\left[\widehat{\epsilon}_{0}(\Lambda), \widehat{A}_{\alpha}\right]_{*}+\Lambda_{\alpha}{ }^{\beta} \widehat{A}_{\beta} \\
\delta_{L} \widehat{A}_{\mu} & =\left[\widehat{\epsilon}_{0}(\Lambda), \widehat{A}_{\mu}\right]_{\star}+\frac{1}{4 i} \partial_{\mu} \Lambda^{\alpha \beta}\left(\widehat{M}_{\alpha \beta}+\frac{1}{2}\left\{\widehat{S}_{\alpha}, \widehat{S}_{\beta}\right\}\right)-\text { h.c. },
\end{aligned}
$$

In obtaining these results, we have used the fact that $\widehat{\epsilon}_{\text {extra }}(\Lambda) \star \widehat{\Phi}-\widehat{\Phi} \star \bar{\pi}\left(\widehat{\epsilon}_{\text {extra }}(\Lambda)\right)=0$ and $\widehat{D}_{\mu} \widehat{\epsilon}_{\text {extra }}(\Lambda)=\partial_{\mu} \Lambda^{\alpha \beta} \frac{1}{8 i}\left\{\widehat{S}_{\alpha}, \widehat{S}_{\beta}\right\}_{\star}$ which follow from $\left[\widehat{S}_{\alpha}, \widehat{f}\right]_{\star}=-2 i \widehat{D}_{\alpha} \widehat{f}$ and the constraints $\widehat{D}_{\alpha} \widehat{\Phi}=0$ and $\widehat{F}_{\alpha \mu}=0$. From (4.11) and (4.13) it follows that the curvature constraints (2.40) and (2.41) are invariant under the local Lorentz transformations

$$
\begin{aligned}
\delta_{L} \Phi & =\left[\epsilon_{0}(\Lambda), \Phi\right]_{\star} \\
\delta_{L} A_{\mu} & =\left[\epsilon_{0}(\Lambda), A_{\mu}\right]_{\star}+\frac{1}{4 i} \partial_{\mu} \Lambda^{\alpha \beta}\left[y_{\alpha} y_{\beta}-4\left(\widehat{A}_{\alpha} \star \widehat{A}_{\beta}\right)_{Z=0}\right]-\text { h.c. },
\end{aligned}
$$

where

$$
\epsilon_{0}(\Lambda)=\frac{1}{4 i} \Lambda^{\alpha \beta} y_{\alpha} y_{\beta}-\text { h.c. . }
$$

The transformation (4.14) has the desired form. However, the last term in the transformation (4.15) shows that the components of $A_{\mu}$ have complicated field dependent Lorentz transformations. We thus have to relate the components of $A_{\mu}$ to the Lorentz connection $\omega_{\mu}^{\alpha \beta}$, which by definition transforms as

$$
\delta_{L} \omega_{\mu}^{\alpha \beta}=\partial_{\mu} \omega_{\mu}^{\alpha \beta}+\Lambda_{\gamma}^{\alpha} \omega_{\mu}^{\gamma \beta}+\Lambda_{\gamma}^{\beta} \omega_{\mu}^{\gamma \alpha}
$$


and a remaining set of Lorentz tensors describing the vierbein $e_{\mu}^{\alpha \dot{\alpha}}$ and the remaining higher spin gauge fields (contained in $W_{\mu}$ below). To do so one observes that from (4.12) and (4.17) it follows that the quantity $\omega_{\mu}+K_{\mu}$, where

$$
\begin{aligned}
\omega_{\mu} & =\frac{1}{4 i} \omega_{\mu}^{\alpha \beta} y_{\alpha} y_{\beta}-\text { h.c. } \\
K_{\mu} & =i \omega_{\mu}^{\alpha \beta}\left(\widehat{A}_{\alpha} \star \widehat{A}_{\beta}\right)_{Z=0}-\text { h.c. }
\end{aligned}
$$

has the following Lorentz transformation

$$
\delta_{L}\left(\omega_{\mu}+K_{\mu}\right)=\left[\epsilon_{0}(\Lambda), \omega_{\mu}+K_{\mu}\right]_{\star}+\frac{1}{4 i} \partial_{\mu} \Lambda^{\alpha \beta}\left[y_{\alpha} y_{\beta}-4\left(\widehat{A}_{\alpha} \star \widehat{A}_{\beta}\right)_{Z=0}\right]-\text { h.c. } .
$$

Thus $\delta_{L}\left(A_{\mu}-\omega_{\mu}-K_{\mu}\right)=\left[\epsilon_{0}(\Lambda), A_{\mu}-\omega_{\mu}-K_{\mu}\right]_{\star}$ which means that $A_{\mu}-\omega_{\mu}-K_{\mu}$ can be expanded in terms of Lorentz tensors. Hence we define

$$
A_{\mu}=E_{\mu}+W_{\mu}+K_{\mu}
$$

where $W_{\mu}$ contains higher spin gauge fields $W_{\mu \alpha_{1} \ldots \alpha_{m} \dot{\alpha}_{1} \ldots \dot{\alpha}_{n}}$ with $m+n=6,10, \ldots$ and $E_{\mu}$ contains the gravitational gauge fields:

$$
E_{\mu}=e_{\mu}+\omega_{\mu}, \quad e_{\mu}=\frac{1}{2 i} e_{\mu}^{\alpha \dot{\alpha}} y_{\alpha} \bar{y}_{\dot{\alpha}}
$$

It follows that under local Lorentz transformations the vierbein and higher spin gauge fields transform as Lorentz tensors:

$$
\begin{aligned}
\delta_{L} e_{\mu}^{\alpha \dot{\alpha}} & =\Lambda_{\gamma}^{\alpha} e_{\mu}^{\gamma \dot{\alpha}}+\Lambda_{\dot{\gamma}}^{\dot{\alpha}} e_{\mu}^{\alpha \dot{\gamma}} \\
\delta_{L} W_{\mu \alpha_{1} \ldots \alpha_{m} \dot{\alpha}_{1} \ldots \dot{\alpha}_{n}} & =-m \Lambda_{\left(\alpha_{1}{ }^{\beta} W_{\left.\mu \alpha_{2} \ldots \alpha_{m}\right) \beta \dot{\alpha}_{1} \ldots \dot{\alpha}_{n}}-n \Lambda_{\left(\dot{\alpha}_{1}\right.}{ }^{\dot{\beta}} W_{\left.\mu \alpha_{1} \ldots \alpha_{m} \dot{\alpha}_{2} \ldots \dot{\alpha}_{n}\right) \dot{\beta}}\right.}
\end{aligned}
$$

Now, we are ready to discuss a weak field expansion scheme which will enable us to probe further the structure of the field equations.

\section{$5 \quad$ Weak Field Expansion}

Let us begin by identifying the physical fields as 


$$
\begin{aligned}
\left.\Phi\right|_{Y=0} & =\phi \\
\left.\left(\sigma^{a}\right)^{\alpha \dot{\alpha}} \frac{\partial}{\partial y^{\alpha}} \frac{\partial}{\partial \bar{y}^{\dot{\alpha}}} E_{\mu}\right|_{Y=0} & =e_{\mu}{ }^{a}, \\
\left.\left(\sigma^{a_{1}}\right)^{\alpha_{1} \dot{\alpha}_{1}} \cdots\left(\sigma^{a_{s-1}}\right)^{\alpha_{s-1} \dot{\alpha}_{s-1}} \frac{\partial}{\partial y^{\alpha_{1}}} \cdots \frac{\partial}{\partial \bar{y}^{\dot{\alpha}_{s-1}}} W_{\mu}\right|_{Y=0} & =W_{\mu}{ }^{a_{1} \ldots a_{s-1}}, \quad s=4,6, \ldots,
\end{aligned}
$$

where $Y=(y, \bar{y})$. All the other components of $\Phi, E_{\mu}$ and $W_{\mu}$, which we collectively refer to as the auxiliary fields, can be eliminated through the use of the constraints (2.40) and (2.41) provided that both $\Phi$ and the spin $s \geq 4$ gauge fields in $W_{\mu}$ are treated as weak fields. Note that $\Phi$ contains the scalar $\phi$

and the non-vanishing spin $s \geq 2$ curvatures (the Weyl tensors) as well as all derivatives of these fields.

In this expansion scheme, the curvature constraints (2.40) and (2.41) up to and including first order in weak fields take the form

$$
\begin{aligned}
\mathcal{R}+F^{(1)}(W) & =-\left(E \star \widehat{E}^{(1)}+\widehat{E}^{(1)} \star E\right)_{Z=0}, \\
d \Phi+E \star \Phi-\Phi \star \bar{\pi}(E) & =0,
\end{aligned}
$$

where

$$
\begin{aligned}
\mathcal{R} & =d E+E \star E \\
F^{(1)}(W) & =D_{E} W=d W+E \star W+W \star E \\
\widehat{E}^{(1)} & =\widehat{L}^{(1)}(E)
\end{aligned}
$$

with $\widehat{L}^{(1)}$ and $E$ defined in (2.38)and (4.22), respectively. Eqs. (2.17) and (5.3) are invariant in the first order under the following gauge transformations ${ }^{5}$

$$
\delta A=d \epsilon+[E, \epsilon]_{\star}, \quad \delta \Phi=0
$$

In the first order, the parameter $b_{1}$ defined in (2.22) and (2.24), which arises on the right hand side of (5.2) through (5.6), (2.38) and (2.33), can be absorbed into a redefinition of $\Phi[16]$. We set $b_{1}=1$ and from (5.2) we obtain

\footnotetext{
${ }^{5}$ The variation of (5.2) and (5.3) is proportional to the Weyl tensor of the gravitational background (which vanishes identically in the AdS background).
} 


$$
\begin{aligned}
& \begin{array}{l}
\text { Gravity } \\
(s=2)
\end{array}: \quad\left\{\begin{array}{l}
\mathcal{R}_{\alpha \beta, \gamma \delta}=\Phi_{\alpha \beta \gamma \delta} \\
\mathcal{R}_{\alpha \beta, \dot{\gamma} \dot{\delta}}=0 \\
\mathcal{R}_{\alpha \beta, \gamma \dot{\delta}}=0
\end{array}\right. \\
& \begin{array}{l}
\text { Higher spins } \\
(s=4,6, \ldots)
\end{array}: \quad\left\{\begin{array}{l}
F_{\alpha \beta, \gamma_{1} \ldots \gamma_{2 s-2}}^{(1)}=\Phi_{\alpha \beta \gamma_{1} \ldots \gamma_{2 s-2}}, \\
F_{\alpha \beta, \gamma_{1} \ldots \gamma_{k} \dot{\gamma}_{k+1} \ldots \dot{\gamma}_{2 s-2}}^{(1)}=0, \quad k=0,1, . ., 2 s-3,
\end{array}\right. \\
& \begin{array}{l}
\text { Scalar } \\
(s=0)
\end{array}: \quad\left\{\begin{array}{l}
\left.\nabla_{\alpha}{ }^{\dot{\alpha}} \Phi_{\beta_{1} \ldots \beta_{m}}{ }^{\dot{\beta}_{1} \ldots \dot{\beta}_{n}}=i \Phi_{\alpha \beta_{1} \ldots \beta_{m}}{ }^{\dot{\alpha} \dot{\beta}_{1} \ldots \dot{\beta}_{n}}-i m n \epsilon_{\alpha\left(\beta_{1}\right.} \epsilon^{\dot{\alpha}\left(\dot{\beta}_{1}\right.} \Phi_{\left.\beta_{2} \ldots \beta_{m}\right)} \dot{\beta}_{2} \ldots \dot{\beta}_{n}\right) \\
|m-n|=0 \bmod 4
\end{array}\right.
\end{aligned}
$$

where $\nabla_{\alpha \dot{\alpha}}=\left(\sigma^{\mu}\right)_{\alpha \dot{\alpha}} \nabla_{\mu}$ is the Lorentz covariant derivative and $F_{\alpha \beta}=\frac{1}{2}\left(\sigma^{\mu \nu}\right)_{\alpha \beta} F_{\mu \nu}$, and $\left(\sigma^{\mu}\right)_{\alpha \dot{\alpha}}=\left(\sigma^{a}\right)_{\alpha \dot{\alpha}} e_{a}{ }^{\mu}$. The Riemann tensor $R_{a}{ }^{b}=d \omega_{a}^{b}+\omega_{a}^{c} \wedge \omega_{c}{ }^{b}$ and torsion $T^{a}=d e^{a}+e^{b} \wedge \omega_{b}^{a}$ arises as

$$
\begin{aligned}
& \mathcal{R}_{\alpha \beta, \gamma \delta}=R_{\alpha \beta, \gamma \delta}+2 \lambda \epsilon_{\alpha(\gamma} \epsilon_{\delta) \beta} \\
& \mathcal{R}_{\alpha \beta, \dot{\gamma} \dot{\delta}}=R_{\alpha \beta, \gamma \delta}, \\
& \mathcal{R}_{\alpha \beta, \gamma \dot{\delta}}=T_{\alpha \beta, \gamma \dot{\delta}} .
\end{aligned}
$$

Equations (5.9) and (5.10) are valid provided that the HS gauge fields $W_{\mu}$ and the curvatures in $\Phi$ are small in units of the AdS radius $R \sim|\lambda|^{-1 / 2}$. Thus, to the leading order in this expansion scheme we can consistently set $W_{\mu}=\phi=0$ in (5.9) and (5.10). Then (5.8) describes the full Einstein equation with a cosmological constant which is proportional to $-|\lambda|$. The spin $s=2$ sector of (5.10), i.e. the components with $m=n \pm 4$, are consistent up to terms which are second order in the spin $s=2$ Weyl tensor $\Phi_{\alpha \beta \gamma \delta}$. Thus, within the expansion scheme (2.28), there is a second expansion scheme in which one expands in powers of $W_{\mu}$ and $\phi$ at fixed, small $\Phi_{\alpha \beta \gamma \delta}$. The basic reason one can expand around a fixed gravitational background is that the $E \star E$ term does not act as a source for higher spins. The second order in the double expansion scheme is obtained by including terms in (2.40) and (2.41) which are quadratic in $\Phi$ and $W_{\mu}$. As a result terms quadratic in $\Phi_{\alpha \beta \gamma \delta}$ and its derivatives appears in the right hand sides of (5.9) so the HS gauge fields become non-zero at second order. Thus, the minimal bosonic HS gauge theory cannot be consistently truncated to pure AdS gravity, though it is possible to expand the theory around a weak gravitational background.

Before proceeding to extract the dynamical field equations to any order, let us summarize the linearized results $[12]$ (see also $[15,16]$ ). The linearized physical field equations are the following components of (5.8), (5.9) and (5.10): 


$$
\begin{aligned}
e_{\alpha_{1}} \dot{\beta} \wedge \mathcal{R}_{\dot{\beta} \dot{\alpha}} & =0, \\
e_{\left(\alpha_{1}{ }^{\dot{\beta}} \wedge F_{\left.\alpha_{2} \ldots \alpha_{s-1}\right) \dot{\beta} \dot{\alpha}_{1} \ldots \dot{\alpha}_{s-1}}^{(1)}\right.} & =0, \\
\left(\nabla^{2}+2\right) \phi & =0 .
\end{aligned}
$$

The last equation is obtained by using the $m=n=0$ and $m=n=1$ components of (5.10). The remaining components of (5.8), (5.9) and (5.10) are used in: a) determining the Weyl tensors $\Phi_{\alpha(2 s)} \equiv \Phi_{\alpha_{1} \ldots \alpha_{2 s}}(s=2,4, \ldots)$ in terms of the curvatures; b) solving for the spin connection $\omega_{\mu}^{\alpha \beta}$ in terms of the vierbein $e_{\mu}^{\alpha \dot{\alpha}}$, and auxiliary higher spin gauge fields $W_{\mu \alpha(m) \dot{\alpha}(n)}$ for $|m-n| \geq 2, m+n=2 s-2$, in terms of the physical fields $W_{\mu \alpha(s-1) \dot{\alpha}(s-1)}(s=4,6, \ldots)$; and c) expressing $\Phi_{\alpha(m) \dot{\alpha}(n)}$ for $m, n>0$ in terms of derivatives of $\phi$ and the Weyl tensors. These steps yield

$$
\begin{aligned}
W_{\alpha \dot{\alpha}, \beta_{1} \ldots \beta_{m} \dot{\beta}_{1} \ldots \dot{\beta}_{n}}= & \frac{2}{m+1} \nabla W_{\dot{\beta}_{1} \dot{\beta}_{2}, \alpha \beta_{1} \ldots \beta_{m} \dot{\alpha} \dot{\beta}_{3} \ldots \dot{\beta}_{n}}+\epsilon_{\dot{\alpha} \dot{\beta}_{1}} \frac{2 n}{n+1}\left[\frac{n-1}{m+n+2} \nabla W_{\dot{\beta}_{2}}{ }^{\dot{\gamma}}{ }_{\alpha \beta_{1} \ldots \beta_{m} \dot{\gamma} \dot{\beta}_{3} \ldots \dot{\beta}_{n}}\right. \\
& \left.+\frac{n+1}{m+n+2} \nabla W_{\left(\alpha, \beta_{1} \ldots \beta_{m}\right) \gamma \dot{\beta}_{2} \ldots \dot{\beta}_{n}}-\frac{m}{(m+1)(m+2)} \epsilon_{\alpha \beta_{1}} \nabla W^{\gamma \delta}{ }_{, \gamma \delta \beta_{2} \ldots \beta_{m} \dot{\beta}_{2} \ldots \dot{\beta}_{n}}\right] \\
& +m \epsilon_{\alpha \beta_{1}} \xi_{\beta_{2} \ldots \beta_{m} \dot{\alpha} \dot{\beta}_{1} \ldots \dot{\beta}_{n}}, \quad n>m \geq 0, \quad m+n=6,10, \ldots(5.17) \\
\Phi_{\alpha_{1} \ldots \alpha_{m} \dot{\alpha}_{1} \ldots \dot{\alpha}_{n}}= & -i \nabla_{\alpha_{1} \dot{\alpha}_{1}} \Phi_{\alpha_{2} \ldots \alpha_{m} \dot{\alpha}_{2} \ldots \dot{\alpha}_{n}},
\end{aligned}
$$

where separate symmetrizations $\left(\alpha_{1} \ldots \alpha_{m}\right)$ and $\left(\dot{\alpha}_{1} \ldots \dot{\alpha}_{n}\right)$, and similarly $\left(\beta_{1} \ldots \beta_{m}\right)$ and $\left(\dot{\beta}_{1} \ldots \dot{\beta}_{n}\right)$ is understood, the derivatives are Lorentz covariant and we have used the notation $W_{\alpha \dot{\alpha}}=\left(\sigma^{\mu}\right)_{\alpha \dot{\alpha}} W_{\mu}$ and

$$
\nabla W_{\alpha \beta, \gamma_{1} \ldots \gamma_{m} \dot{\gamma}_{1} \ldots \dot{\gamma}_{n}} \equiv \frac{1}{2}\left(\sigma^{\mu \nu}\right)_{\alpha \beta} \nabla_{\mu} W_{\nu \gamma_{1} \ldots \gamma_{m} \dot{\gamma}_{1} \ldots \dot{\gamma}_{n}}
$$

In $(5.17)$ the components $\xi_{\beta 1 \ldots \beta_{m-1} \dot{\beta}_{1} \ldots \dot{\beta}_{n+1}}$ are not fixed by the generalized torsion constraints. However, from the component form of the linearized symmetries (5.7),

$\delta W_{\mu \alpha_{1} \ldots \alpha_{m} \dot{\alpha}_{1} \ldots \dot{\alpha}_{n}}=\nabla_{\mu} \epsilon_{\alpha_{1} \ldots \alpha_{m} \dot{\alpha}_{1} \ldots \dot{\alpha}_{n}}+m\left(\sigma_{\mu}\right)_{\left(\alpha_{1}\right.}{ }^{\dot{\beta}} \epsilon_{\left.\alpha_{2} \ldots \alpha_{m}\right) \dot{\alpha}_{1} \ldots \dot{\alpha}_{n} \dot{\beta}}+n\left(\bar{\sigma}_{\mu}\right)_{\left(\dot{\alpha}_{1}{ }^{\beta} \epsilon_{\left.\alpha_{1} \ldots \alpha_{m} \beta \dot{\alpha}_{2} \ldots \dot{\alpha}_{2}\right)} .\right.}$.

it follows that the auxiliary gauge parameter $\epsilon_{\alpha(m) \dot{\alpha}(n)}$ with $|m-n|=4,6, \ldots$ acts by shifting the undetermined component $\xi_{\alpha(m) \dot{\alpha}(n)}$. The undetermined components are 
therefore gauge artifacts which can be eliminated by going to a physical gauge. For example, we can impose

$$
\left(\sigma^{\mu}\right)_{\left(\alpha_{1}\right.}{ }^{\dot{\beta}} W_{\left.\mu \alpha_{2} \ldots \alpha_{m}\right) \dot{\alpha}_{1} \ldots \dot{\alpha}_{n} \dot{\beta}}=0, \quad m-n=4,6, \ldots .
$$

The first order physical field equations (5.14)-(5.16) are invariant under the remaining generalized Lorentz rotations and generalized translations with parameters $\epsilon_{\alpha(s-2) \dot{\alpha}(s)}$ and $\epsilon_{\alpha(s-1) \dot{\alpha}(s-1)}$, respectively $(s=2,4, \ldots)$. Some of these gauge symmetries can be used to recover Fronsdal's formulation of free massless higher spin fields in terms of doubly traceless symmetric tensors $(s=4,6, \ldots)$

$$
\begin{aligned}
h_{\mu_{1} \ldots \mu_{s}} & =\left(\sigma_{\mu_{2}}\right)^{\alpha_{1} \dot{\alpha}_{1}} \cdots\left(\sigma_{\mu_{2}}\right)^{\alpha_{s-1} \dot{\alpha}_{s-1}} W_{\mu_{1} \alpha_{1} \ldots \alpha_{s-1} \dot{\alpha}_{1} \ldots \dot{\alpha}_{s-1}} \\
g^{\mu \nu} g^{\rho \sigma} h_{\mu \nu \rho \sigma \lambda(s-4)} & =0
\end{aligned}
$$

obeying field equations which are invariant under the remaining translation-like symmetries

$$
\delta h_{\mu_{1} \ldots \mu_{s}}=\nabla_{\left(\mu_{1}\right.} \epsilon_{\left.\mu_{2} \ldots \mu_{s}\right)}-\text { double traces }
$$

These symmetries remove the single-traces as well as the time-like and longitudinal modes, leading to two physical transverse spin $s$ modes with energy $E_{0}=s+1$ for $s=4, \ldots$. Together with the physical scalar with energy $E_{0}=1$, which is described by (5.16), and the graviton this results in a spectrum isomorphic to the symmetric tensor product of two scalar $S O(3,2)$ singletons.

In going beyond first order, each component of the curvature constraints (2.40) and (2.41) continues to serve the purpose it did in the first order analysis. For example, a first order generalized torsion constraint, from which an auxiliary gauge field is eliminated in the leading order, plays the same role in the full theory, though the elimination procedure must of course be done perturbatively (see (5.29) and (5.30) below). Similarly, the full field equations are contained in the same components of (2.40) and (2.41) as the first order field equations (5.14) and (5.16), i.e. they are given by

$$
\begin{aligned}
& e_{\alpha}^{\dot{\beta}} \wedge \mathcal{R}_{\dot{\beta} \dot{\alpha}}=e_{\alpha}^{\dot{\beta}} \wedge J_{\dot{\beta} \dot{\alpha}} \\
& e_{\left(\alpha_{1}\right.}^{\dot{\beta}} \wedge F_{\left.\alpha_{2} \ldots \alpha_{s-1}\right) \dot{\beta} \dot{\alpha}_{1} \ldots \dot{\alpha}_{s-1}}^{(1)}=e_{\left(\alpha_{1}\right.} \dot{\beta} \wedge J_{\left.\alpha_{2} \ldots \alpha_{s-1}\right) \dot{\beta} \dot{\alpha}_{1} \ldots \dot{\alpha}_{s-1}} \\
& \left(\nabla^{2}+2\right) \phi=\nabla^{\mu} P_{\mu}-\frac{i}{2}\left(\sigma^{\mu}\right)^{\alpha \dot{\alpha}} P_{\mu, \alpha \dot{\alpha}}
\end{aligned}
$$


where the two-form $J=J^{(2)}+J^{(3)}+\cdots$ and the one-form $P=P^{(2)}+P^{(3)}+\cdots$, which contain the interactions, are given by

$$
\begin{aligned}
J & =-D_{E} K-(W+K) \star(W+K)-\sum_{n=2}^{\infty} \sum_{j=0}^{n}\left(\widehat{A}^{(j)} \star \widehat{A}^{(n-j)}\right)_{Z=0} \\
P & =\Phi \star \bar{\pi}(W+K)-(W+K) \star \Phi+\sum_{n=2}^{\infty} \sum_{j=1}^{n}\left(\widehat{\Phi}^{(j)} \star \bar{\pi}\left(\widehat{A}^{(n-j)}\right)-\widehat{A}^{(n-j)} \star \widehat{\Phi}^{(j)}\right)_{Z=0},
\end{aligned}
$$

where $D_{E} K=d K+E * K+K * E$ and the quantity $K=d x^{\mu} K_{\mu}$ is defined in (4.19) and it has the expansion $K=K^{(2)}+K^{(3)}+\cdots$. The contracted curvatures in the right hand sides of (5.25) and (5.26) contain the vierbein $e_{\mu \alpha \dot{\alpha}}$ and its higher spin generalizations $W_{\mu \alpha_{1} \ldots \alpha_{s-1} \dot{\alpha}_{1} \ldots \dot{\alpha}_{s-1}}$ and first order derivatives of the Lorentz connection $\omega_{\mu \alpha \beta}$ and its higher spin generalizations $W_{\mu \alpha_{1} \ldots \alpha_{s-2} \dot{\alpha}_{1} \ldots \dot{\alpha}_{s}}$.

The general solutions for the auxiliary fields are modified as

$$
\begin{aligned}
W_{\alpha \dot{\alpha}, \beta_{1} \ldots \beta_{m} \dot{\beta}_{1} \ldots \dot{\beta}_{n}}= & \frac{2}{m+1} \widehat{\nabla} W_{\dot{\beta}_{1} \dot{\beta}_{2}, \alpha \beta_{1} \ldots \beta_{m} \dot{\alpha} \dot{\beta}_{3} \ldots \dot{\beta}_{n}}+\epsilon_{\dot{\alpha} \dot{\beta}_{1}} \frac{2 n}{n+1}\left[\frac{n-1}{m+n+2} \widehat{\nabla} W_{\dot{\beta}_{2}}{ }_{, \alpha \beta_{1} \ldots \beta_{m} \dot{\gamma} \dot{\beta}_{3} \ldots \dot{\beta}_{n}}\right. \\
& \left.+\frac{n+1}{m+n+2} \widehat{\nabla} W_{\left(\alpha, \beta_{1} \ldots \beta_{m}\right) \gamma \dot{\beta}_{2} \ldots \dot{\beta}_{n}}-\frac{m}{(m+1)(m+2)} \epsilon_{\alpha \beta_{1}} \widehat{\nabla} W^{\gamma \delta}{ }_{, \gamma \delta \beta_{2} \ldots \beta_{m} \dot{\beta}_{2} \ldots \dot{\beta}_{n}}\right] \\
& +m \epsilon_{\alpha \beta_{1}} \xi_{\beta 2 \ldots \beta_{m} \dot{\alpha} \dot{\beta}_{1} \ldots \dot{\beta}_{n}}, \quad n>m \geq 0, \\
\Phi_{\alpha_{1} \ldots \alpha_{m} \dot{\alpha}_{1} \ldots \dot{\alpha}_{n}}= & -i \widehat{\nabla}_{\alpha_{1} \dot{\alpha}_{1}} \Phi_{\alpha_{2} \ldots \alpha_{m} \dot{\alpha}_{2} \ldots \dot{\alpha}_{n}},
\end{aligned}
$$

where the modified covariant derivatives are defined by

$$
\begin{aligned}
& \widehat{\nabla} W_{\alpha \beta, \gamma_{1} \ldots \gamma_{m} \dot{\gamma}_{1} \ldots \dot{\gamma}_{n}}=\frac{1}{2}\left(\sigma^{\mu \nu}\right)_{\alpha \beta}\left(\nabla_{\mu} W_{\nu \gamma_{1} \ldots \gamma_{m} \dot{\gamma}_{1} \ldots \dot{\gamma}_{n}}-\frac{1}{2} J_{\mu \nu \gamma_{1} \ldots \gamma_{m} \dot{\gamma}_{1} \ldots \dot{\gamma}_{n}}\right) \\
& \widehat{\nabla}_{\alpha_{1} \dot{\alpha}_{1}} \Phi_{\alpha_{2} \ldots \alpha_{m} \dot{\alpha}_{2} \ldots \dot{\alpha}_{n}}=\left(\nabla_{\alpha_{1} \dot{\alpha}_{1}} \Phi_{\alpha_{2} \ldots \alpha_{m} \dot{\alpha}_{2} \ldots \dot{\alpha}_{n}}-P_{\alpha_{1} \dot{\alpha}_{1}, \alpha_{2} \ldots \alpha_{m} \dot{\alpha}_{2} \ldots \dot{\alpha}_{n}}\right)
\end{aligned}
$$

Since $J_{\mu \nu}$ and $P_{\mu}$ depend on the auxiliary fields, eqs. (5.29) and (5.30) must be iterated within the curvature expansion scheme. This leads to explicit expressions of all auxiliary components of $W_{\mu}$ and $\Phi$ in terms of the remaining physical fields (5.1) to any desired order. Inserting these expressions into (5.25), (5.26) and (5.27) we find the physical field equations. 
The non-covariant contributions to $J_{\mu \nu}$ and $P_{\mu}$ from the Lorentz connection $\omega_{\mu}$ must cancel, since the full curvature constraints as well as the quantities $\mathcal{R}_{\mu \nu}, F^{(1)}(W)_{\mu \nu}$ and $\nabla_{\mu} \Phi$ are Lorentz covariant. Thus the $\omega_{\mu}$ dependence in $P_{\mu}$ can be dropped immediately, while in $J_{\mu \nu}$ we first covariantize $D_{E} K$ by writing it as

$$
D_{E} K=i R^{\alpha \beta}\left(\widehat{A}_{\alpha} \star \widehat{A}_{\beta}\right)_{Z=0} \text { - h.c. }+ \text { non- covariant } \omega \text { dependence },
$$

where $R_{\alpha \beta}$ is the Riemann curvature two-form. From the manifest Lorentz invariance it follows that the non-covariant $\omega$ dependence in $J$ and $P$ must cancel. Thus:

$$
\begin{aligned}
J= & -i\left(R^{\alpha \beta} \widehat{A}_{\alpha} \star \widehat{A}_{\beta}+R^{\dot{\alpha} \dot{\beta}} \widehat{A}_{\dot{\alpha}} \star \widehat{A}_{\dot{\beta}}\right)_{Z=0}-W \star W \\
& -\sum_{n=2}^{\infty} \sum_{j=0}^{n}\left(\left(\widehat{e}^{(j)}+\widehat{W}^{(j)}\right) \star\left(\widehat{e}^{(n-j)}+\widehat{W}^{(n-j)}\right)\right)_{Z=0}, \\
P= & \Phi \star \bar{\pi}(W)-(W) \star \Phi \\
& +\sum_{n=2}^{\infty} \sum_{j=1}^{n}\left(\widehat{\Phi}^{(j)} \star \bar{\pi}\left(\widehat{e}^{(n-j)}+\widehat{W}^{(n-j)}\right)-\left(\widehat{e}^{(n-j)}+\widehat{W}^{(n-j)}\right) \star \widehat{\Phi}^{(j)}\right)_{Z=0}
\end{aligned}
$$

where $R_{\dot{\alpha} \dot{\beta}}=\left(R_{\alpha \beta}\right)^{\dagger}$.

It is straightforward to modify the above formulae such that the gravitational connections $e$ and $\omega$ become linearized around an AdS-like vacuum $\Omega$ obeying $d \Omega+\Omega \star \Omega=0$. The Lorentz covariant derivative $\nabla_{\mu}$ then becomes background Lorentz covariant and $e$ splits into AdS background plus a fluctuation part which can be accounted for by including also spin $s=2$ fluctuations in $W$.

The physical field equations (5.25) and (5.27) are manifestly invariant under the local Lorentz transformations (4.17) and (4.23) and (4.24). They are also invariant under diffeomorphisms and HS gauge symmetries. In general, a set of integrable constraints

$$
R^{I} \equiv d C^{I}+F^{I}\left(C^{J}\right)=0, \quad \frac{\partial F^{I}}{\partial C^{J}} \wedge F^{J}=0,
$$

where $C^{I}$ are $n_{I}$-forms, have the gauge symmetry

$$
\delta C^{I}= \begin{cases}d \epsilon^{I}+\frac{\partial F^{I}}{\partial C^{J}} \wedge \epsilon^{J}, & n_{I} \geq 1 \\ \frac{\partial F^{I}}{\partial C^{J}} \wedge \epsilon^{J}, & n_{I}=0\end{cases}
$$


Diffeomorphisms, $\delta W^{I}=\left\{d, i_{\xi}\right\} W^{I}$ where $\xi=d x^{\mu} \xi_{\mu}$, are incorporated into the gauge group as field dependent transformations with gauge parameter $\epsilon^{I}=i_{\xi} W^{I}$. To apply these formulae to the full constraints $(2.40)$ and $(2.41)$ we set $C^{I}=(e, \omega, W ; \Phi)$. We then find that the gauge transformations associated with $e$ and $W$ are

$$
\begin{aligned}
\delta(e+\omega+W) & =\nabla \epsilon+[e+W, \epsilon]_{\star}+\sum_{n=2}^{\infty} \sum_{j=0}^{n}\left(\left[\widehat{e}^{(j)}+\widehat{W}^{(j)}, \widehat{\epsilon}^{(n-j)}\right]_{\star}\right)_{Z=0}, \\
\delta \Phi & =\Phi \star \bar{\pi}(\epsilon)-\epsilon \star \Phi+\sum_{n=2}^{\infty} \sum_{j=1}^{n}\left(\widehat{\Phi}^{(j)} \star \bar{\pi}\left(\widehat{\epsilon}^{(n-j)}-\widehat{\epsilon}^{(n-j)} \star \widehat{\Phi}^{(j)}\right)\right)_{Z=0}(5.39
\end{aligned}
$$

where $\epsilon$ is either an ordinary translation, $\epsilon_{\alpha \dot{\alpha}} P^{\alpha \dot{\alpha}}$ or a HS gauge parameter, $\epsilon_{\alpha(m) \dot{\alpha}(n)}$, $m+n=6,10, \ldots$. On general grounds, the above transformations must agree with the form of the residual $Z$-space transformations discussed at the end of Section 2. As in the first order, the auxiliary gauge symmetries with parameters $\epsilon_{\alpha(m) \dot{\alpha}(n)},|m-n|=$ $4,6, \ldots$ act by curvature corrected shifts of the $\xi$-components in $(5.29)$, which can be used to impose a physical gauge, such as (5.21). In general, there may of course be other physical gauge choices which lead to further simplification. The remaining gauge symmetries, i.e. the local translations and Lorentz transformations and their higher spin generalizations with $|m-n|=0,2$, respectively, act as symmetries of the dynamical field equations in (5.25), (5.26) and (5.27).

\section{Closer Look at the Quadratic Terms in The Field Equations}

In this section, we collect the results of the previous section for the important special case of the quadratic terms in the field equations. The field equations up to quadratic order in weak field approximation are given by

$$
\begin{aligned}
\left(\nabla^{2}+2\right) \phi & =\left(\nabla^{\mu} P_{\mu}^{(2)}-\frac{i}{2}\left(\sigma^{\mu}\right)^{\alpha \dot{\alpha}} \frac{\partial}{\partial y^{\alpha}} \frac{\partial}{\partial \bar{y}^{\dot{\alpha}}} P_{\mu}^{(2)}\right)_{Y=0}, \\
\left(\sigma^{\mu \nu \rho}\right)_{\alpha}^{\dot{\beta}} \mathcal{R}_{\nu \rho \dot{\beta} \dot{\gamma}} & =\left(\sigma^{\mu \nu \rho}\right)_{\alpha}^{\dot{\beta}}\left(\frac{\partial}{\partial \bar{y}^{\dot{\beta}}} \frac{\partial}{\partial \bar{y}^{\dot{\gamma}}} J_{\nu \rho}^{(2)}\right)_{Y=0}, \\
\left(\sigma^{\mu \nu \rho}\right)_{\left(\alpha_{1}\right.}^{\dot{\beta}} F_{\left.\nu \rho \alpha_{2} \ldots \alpha_{s-1}\right) \dot{\beta} \dot{\gamma} \dot{\alpha}_{2} \ldots \dot{\alpha}_{s-1}}^{(1)} & \left.=\left(\sigma^{\mu \nu \rho}\right)_{\left(\alpha_{1}\right.}^{\dot{\beta}}\left(\frac{\partial}{\partial y^{\alpha_{2}}} \cdots \frac{\partial}{\partial y^{\left.\alpha_{s-1}\right)}} \frac{\partial}{\partial \bar{y}^{\dot{\beta}}} \cdots \frac{\partial}{\partial \bar{y}^{\dot{\alpha}_{s-1}}} J_{\nu \rho}^{(2)}\right)_{Y=0} 6.3\right)
\end{aligned}
$$


where $\mathcal{R}_{\mu \nu \dot{\alpha} \dot{\beta}} \equiv F_{\mu \nu \dot{\alpha} \dot{\beta}}$ is the (self-dual part of) the $A d S_{4}$ valued Riemann curvature, while the curvature associated with spin $s=4,6,8, .$. fields is defined as

$$
\begin{aligned}
F_{\nu \rho \alpha_{2} \ldots \alpha_{s-1} \dot{\beta} \dot{\gamma} \dot{\alpha}_{2} \ldots \dot{\alpha}_{s-1}}^{(1)}= & 2 \nabla_{[\nu} W_{\rho] \alpha_{2} \ldots \alpha_{s-1} \dot{\beta} \dot{\gamma} \dot{\alpha}_{2} \ldots \dot{\alpha}_{s-1}} \\
& -(s-2)\left(\sigma_{\nu \rho} \sigma_{\mu}\right)_{\left(\alpha_{2}\right.}{ }^{\delta} W_{\left.\mu \alpha_{3} \ldots \alpha_{s-1}\right) \delta \dot{\beta} \dot{\gamma} \dot{\delta} \dot{\alpha} \dot{\alpha}_{2} \ldots \dot{\alpha}_{s-1}} \\
& -s\left(\sigma_{\mu} \sigma_{\nu \rho}\right)_{(\dot{\beta}}{ }^{\gamma} W_{\left.\mu \gamma \alpha_{2} \alpha_{3} \ldots \alpha_{s-1} \dot{\gamma} \dot{\alpha}_{2} \ldots \dot{\alpha}_{s-1}\right)}
\end{aligned}
$$

The covariant derivatives in (6.1) and (6.4) are with respect to the Lorentz connection $\omega$. Further definitions are

$$
\begin{aligned}
P_{\mu}^{(2)}= & \Phi \star \bar{\pi}\left(W_{\mu}\right)-W_{\mu} \star \Phi \\
& +\left(\Phi \star \bar{\pi}\left(\widehat{e}_{\mu}^{(1)}\right)-\widehat{e}_{\mu}^{(1)} \star \Phi+\widehat{\Phi}^{(2)} \star \bar{\pi}\left(e_{\mu}\right)-e_{\mu} \star \widehat{\Phi}^{(2)}\right)_{Z=0} \\
J_{\mu \nu}^{(2)}= & -\left[\left(\widehat{e}_{\mu}^{(1)} \star \widehat{e}_{\nu}^{(1)}+\left\{e_{\mu}, \widehat{e}_{\nu}^{(2)}\right\}_{\star}+\left\{e_{\mu}, \widehat{W}_{\nu}^{(1)}\right\}_{\star}+\left\{W_{\mu}, \widehat{e}_{\nu}^{(1)}\right\}_{\star}\right)_{Z=0}\right. \\
& \left.+\left(i R_{\mu \nu}{ }^{\alpha \beta} \widehat{A}_{\alpha}^{(1)} \star \widehat{A}_{\beta}^{(1)}+\text { h.c. }\right)_{Z=0}+W_{\mu} \star W_{\nu}\right]+[\mu \leftrightarrow \nu],
\end{aligned}
$$

where the hatted quantities are defined as

$$
\begin{aligned}
\widehat{A}_{\alpha}^{(1)}= & -\frac{i}{2} z_{\alpha} \int_{0}^{1} t d t b_{1} \Phi(-t z, \bar{y}) \kappa(t z, y) \\
\widehat{A}_{\alpha}^{(2)}= & z_{\alpha} \int_{0}^{1} t d t\left(\widehat{A}^{(1) \beta} \star \widehat{A}_{\beta}^{(1)}\right)_{z \rightarrow t z, \bar{z} \rightarrow t \bar{z}} \\
& +\bar{z}^{\dot{\beta}} \int_{0}^{1} t d t\left[\widehat{A}_{\alpha}^{(1)}, \widehat{A}_{\dot{\beta}}^{(1)}\right]_{z \rightarrow t z, \bar{z} \rightarrow t \bar{z}} \\
\widehat{W}_{\mu}^{(1)}= & \widehat{L}^{(1)}\left(W_{\mu}\right) \\
= & -i \int_{0}^{1} \frac{d t}{t}\left(\left[\frac{\partial W_{\mu}}{\partial y^{\alpha}}, \widehat{A}^{\alpha(1)}\right]_{*}+\left[\widehat{A}^{\dot{\alpha}(1)}, \frac{\partial W_{\mu}}{\partial \bar{y}^{\dot{\alpha}}}\right]_{*}\right)_{z \rightarrow t z, \bar{z} \rightarrow t \bar{z}} \\
\widehat{\Phi}^{(2)}= & z^{\alpha} \int_{0}^{1} d t\left[\Phi \star \bar{\pi}\left(\widehat{A}_{\alpha}^{(1)}\right)-\widehat{A}_{\alpha}^{(1)} \star \Phi\right]_{t \rightarrow t z, \bar{z} \rightarrow t \bar{z}}
\end{aligned}
$$




$$
\begin{aligned}
& +\bar{z}^{\dot{\alpha}} \int_{0}^{1} d t\left[\Phi \star \pi\left(\widehat{A}_{\dot{\alpha}}^{(1)}\right)-\widehat{A}_{\dot{\alpha}}^{(1)} \star \Phi\right]_{t \rightarrow t z, \bar{z} \rightarrow t \bar{z}} \\
\widehat{e}_{\mu}^{(1)}= & \widehat{L}^{(1)}\left(e_{\mu}\right) \\
= & -i e_{\mu}^{\alpha \dot{\alpha}} \int_{0}^{1} \frac{d t}{t}\left(\left[\bar{y}_{\dot{\alpha}}, \widehat{A}_{\alpha}^{(1)}\right]_{*}+\left[\widehat{A}_{\dot{\alpha}}^{(1)}, y_{\alpha}\right]_{*}\right)_{z \rightarrow t z, \bar{z} \rightarrow t \bar{z}} \\
\widehat{e}_{\mu}^{(2)=} & \left(\widehat{L}^{(2)}+\widehat{L}^{(1)} \widehat{L}^{(1)}\right)\left(e_{\mu}\right) \\
= & -i e_{\mu}^{\alpha \dot{\alpha}} \int_{0}^{1} \frac{d t}{t}\left(\left[\bar{y}_{\dot{\alpha}}, \widehat{A}_{\alpha}^{(2)}\right]_{*}+\left[\widehat{A}_{\dot{\alpha}}^{(2)}, y_{\alpha}\right]_{*}\right)_{z \rightarrow t z, \bar{z} \rightarrow t \bar{z}} \\
& -e_{\mu}^{\alpha \dot{\alpha}} \int_{0}^{1} \frac{d t}{t} \int_{0}^{1} \frac{d t^{\prime}}{t^{\prime}}\left[\widehat{A}^{\beta(1)} \star\left(\frac{\partial}{\partial z^{\beta}}-\frac{\partial}{\partial y^{\beta}}\right)\left(\left[\bar{y}_{\dot{\alpha}}, \widehat{A}_{\alpha}^{(1)}\right]_{*}+\left[\widehat{A}_{\dot{\alpha}}^{(1)}, y_{\alpha}\right]_{*}\right)_{z \rightarrow t^{\prime} z, \bar{z} \rightarrow t^{\prime} \bar{z}}\right. \\
& +\left(\frac{\partial}{\partial z^{\beta}}+\frac{\partial}{\partial y^{\beta}}\right)\left(\left[\bar{y}_{\dot{\alpha}}, \widehat{A}_{\alpha}^{(1)}\right]_{*}+\left[\widehat{A}_{\dot{\alpha}}^{(1)}, y_{\alpha}\right]_{*}\right)_{z \rightarrow t^{\prime} z, \bar{z} \rightarrow t^{\prime} \bar{z}} \star \widehat{A}^{\beta(1)} \\
& \left.+\left(\frac{\partial}{\partial \bar{z}^{\dot{\beta}}}-\frac{\partial}{\partial \bar{y}^{\dot{\beta}}}\right)\left(\left[\bar{y}_{\dot{\alpha}}, \widehat{A}_{\alpha}^{(1)}\right]_{*}+\left[\widehat{A}_{\dot{\alpha}}^{(1)}, y_{\alpha}\right]_{*}\right)_{z \rightarrow t^{\prime} z, \bar{z} \rightarrow t^{\prime} \bar{z}} \star \widehat{A}^{\dot{\beta}(1)}\right]_{z \rightarrow t z, \bar{z} \rightarrow t \bar{z}}
\end{aligned}
$$

where the constant $b_{1}$ in (6.7) is defined by (2.22) and (2.24). In the above formulae, the replacement $(z, \bar{z}) \rightarrow(t z, t \bar{z})$ in a quantity must be performed after the quantity has been written in Weyl ordered form i.e. after the $\star$ products defining the quantity has been performed.

A closer look at the field equations presented above shows that:

- The $z$-dependence of all the fields involved is either a simple dependence which is explicitly shown or it arises through dressing via the action of the $\widehat{L}$ operator. All in all, the above results are explicit and the remaining task is the evaluation of certain star products (involving finite order differentiations in $y$ and $z$ ) and some elementary parameter integrals. The results of these computations will be given elsewhere [14].

- The field equation (6.2) for the graviton can be rewritten as ${ }^{6}$

\footnotetext{
${ }^{6}$ We have set the AdS radius $R=1$ but it is straightforward to re-introduce $R$ by dimensional analysis in which the master 0 -form and the master 1 -form fields are dimensionless.
} 


$$
R_{\mu \nu}(\omega)-g_{\mu \nu}=\left[\left(\sigma_{\mu}{ }^{\lambda}\right)^{\alpha \beta}\left(\frac{\partial}{\partial y^{\alpha}} \frac{\partial}{\partial y^{\beta}} J_{\lambda \nu}^{(2)}\right)_{Y=0}+(\mu \leftrightarrow \nu)+\text { h.c. }\right],
$$

where $R_{\mu \nu}(\omega)$ is the Ricci tensor obtained from the Riemann tensor associated with the Lorentz connection $\omega_{\mu}$. It is important to note that this connection contains torsion as can be seen from (5.29) and (5.31) which for $m=0, n=2$ give

$$
\omega_{\mu}^{a b}=\omega_{\mu}^{a b}(e)+\kappa_{\mu}^{a b}
$$

where $\kappa_{\mu}^{a b}$ is the con-torsion tensor related to the torsion tensor $T_{\mu \nu}{ }^{a}$ as

$$
\kappa_{\mu}^{a b}=T_{\mu}^{a b}-T_{\mu}^{b a}+T_{\mu}^{a b},
$$

where

$$
T_{\mu \nu}^{a}=\left(\sigma^{a}\right)_{\alpha \dot{\beta}}\left(\frac{\partial}{\partial y_{\alpha}} \frac{\partial}{\partial \bar{y}_{\dot{\beta}}} J_{\mu \nu}^{(2)}\right)_{Y=0} .
$$

- In the final form of the field equations, the auxiliary fields arise both in the master scalar field $\Phi$ as well as the master gauge field $A_{\mu}$. Their solution given in (5.30) and (5.29) in terms of the physical fields are to be used in these equations. It is through the elimination of these auxiliary fields that higher derivative interactions arise. In particular, in a given spin sector, the auxiliary fields are $W_{\mu \alpha_{1} \ldots \alpha_{k} \dot{\alpha}_{k+1} \ldots \dot{\alpha}_{2 s-2}}$ with $k=0,1, \ldots, s / 2$ and they are related to the physical fields $W_{\mu \alpha(s-1) \dot{\alpha}(s-1)}$ schematically as

$$
W_{\mu \alpha(m) \dot{\alpha}(n)} \sim \partial^{|m-n| / 2} W_{\mu \alpha(s-1) \dot{\alpha}(s-1)}, \quad m+n=2 s-2 .
$$

Similarly, the components $\Phi_{\alpha(m) \dot{\alpha}(n)}$ of the master scalar field are related to the Weyl tensors which are purely chiral, their derivatives as well as the derivatives of the scalar as (taking $m>n$ without loss of generality)

$$
\begin{aligned}
\Phi_{\alpha(m) \dot{\alpha}(m)} & \sim \partial^{m} \phi \\
\Phi_{\alpha(m) \dot{\alpha}(n)} & \sim \partial^{(m-n) / 2} \Phi_{\alpha(m-n)}, \quad m-n=0 \bmod 4 .
\end{aligned}
$$

The preliminary results of [14] indicate that the contribution from the physical scalar field to the stress energy tensor in (6.13) involves derivatives of the scalar to arbitrary order period. Whether the higher orders can be absorbed into a field redefinition remains to be seen. 
- The field equations (5.27) and (6.3) are invariant under generalized Lorentz rotations and translations. Their action, including the compensating gauge transformations due to (5.21), is given by (5.38) and (5.39), where one of course retains only terms up to quadratic in order in $\epsilon, W$ and $\Phi$.

\section{Discussion}

In this paper we have studied the structure of a minimal bosonic HS gauge theory in four dimensions. We have presented in considerable detail an iterative procedure for determining the field equations to any desired order in weak fields, which are the physical scalar and the higher spin gauge fields as well as arbitrary derivatives of the physical fields, including the spin 2 Weyl tensor and its derivatives. We note that the vierbein is not expanded around any fixed background and the field equations are manifestly reparametrization and local Lorentz invariant.

We emphasize that the building blocks of the theory are the master 0 -form $\hat{\Phi}$ containing the physical scalar, the Weyl tensors and derivatives, and a master 1 -form $\hat{A}$ containing the physical spin $s=2,4, \ldots, \infty$ fields and auxiliary fields which can be solved for in terms of the physical fields and their derivatives. The central equation that describes the theory is (see Section 2)

$$
d \widehat{\Phi}+\widehat{A} \star \widehat{\Phi}-\widehat{\Phi} \star \bar{\pi}(\widehat{A})=0
$$

The integrability of this equation naturally leads to the second master equation (modulo the generalization involving $\mathcal{V}(X)$ discussed in Section 2)

$$
d \widehat{A}+\widehat{A} \star \widehat{A}=\frac{i}{4} d z^{\alpha} \wedge d z_{\alpha} \mathcal{V}(\widehat{\Phi} \star \kappa)-\text { h.c. },
$$

which together with (7.1) form a complete set of constraints defining the theory. For weak $\widehat{\Phi}$, eqs. (7.1) and (7.2) are equivalent to the constraints (2.40) and (2.41) in ordinary spacetime, which have been the main focus of the discussion in this paper. It is worth pointing out, however, that (7.1) and (7.2) imply that $\widehat{A}_{\mu}$ is locally a pure gauge field. Thus, in a neighborhood of a spacetime point, $p$ say, (7.1) and (7.2), and therefore (2.40) and (2.41), are equivalent to $\left.\widehat{F}_{\alpha \dot{\alpha}}\right|_{p}=0,\left.\left(\widehat{F}_{\alpha \beta}+\frac{i}{2} \epsilon_{\alpha \beta} \mathcal{V}(\widehat{\Phi} \star \kappa)\right)\right|_{p}=0$ and $\left.\widehat{\Phi}\right|_{p}=0$. The use of this in the context of the AdS/HS gauge theory correspondence remains to be examined.

The master scalar field is essential for the consistency of the theory in that it contains the physical scalar field which is required for a physical spectrum that fits into an UIR of the HS symmetry group that underlies the theory. At the same time, the presence 
of the master scalar field complicates the problem of finding an action. Indeed, in the absence of the scalar, one can write down an action of the type $S=\int d^{4} x \operatorname{Tr} F \star \wedge F$, where $\mathrm{Tr}$ is the trace over the HS algebra [13]. However, since this action does not contain the physical scalar field it does not describe a consistent HS gauge theory. This problem does not arise in 3D, where actions for higher spin gauge theories including their matter couplings have been constructed; see, for example, [29, 13].

The master field equations (7.1) and (7.2) are deceptively simple looking. They embody equations that contain curvatures of the physical fields and their derivatives to arbitrarily high order, which can be obtained in a weak field expansion by the iterative procedure explained in the paper. A glance at the resulting equations up to second order in weak fields given in eqs. (6.1) and (6.3) reveal that the detailed form of the equations is very complicated. Considerable may be achieved by working in a first order formalism where the auxiliary fields are treated as independent fields. The fact that there exist composite operators on the boundary corresponding to the auxiliary fields $[22]$ is encouraging in this respect.

The results of this paper can easily be carried over to the HS gauge theory extension of the $D=4, \mathcal{N}=8$ supergravity. This theory admits the minimal bosonic theory as a consistent truncation. A search for a holographic description of the $\mathcal{N}=8$ theory is the most interesting one as it is naturally related to $M$ theory on $A d S_{4} \times S^{7}$. Nonetheless, some basic aspects of holography in this context can already be glimpsed from a study of the minimal bosonic model. In that case, a relevant boundary field theory to consider is scalar singleton $W$ in the adjoint representation of $S U(N)$ for large $N$. Bilinear operators corresponding to the physical spectrum of the bulk HS gauge theory can be built out of two such singletons and their derivatives. The operators corresponding to spin $s=2,4, \ldots$ fields are conserved tensors, while the physical scalar couples to $: \operatorname{tr} W^{2}:$.

The free singleton theory also contains composite operators which are cubic or higher order in the singleton field $W$ and that couple to massive bulk fields. It has been proposed in [10] that in the $\mathcal{N}=8$ theory the massless bulk fields decouple consistently from the massive bulk fields in the leading order in the $1 / N$ expansion. This is crucial for the massless HS theory to make sense as a limit of M theory since there is no massgap between the massless and the massive fields. The truncation to the massless sector can be tested since it implies that there exists a choice of interaction $\mathcal{V}(X)$ given in (7.2) such that bulk theory reproduces the correlators of the bilinear operators on the boundary. Moreover, this test can be performed at the level of the massless minimal bosonic theory [14], which is a consistent truncation of the massless $\mathcal{N}=8$ theory.

\section{Acknowledgements}

P.S. is thankful to U. Danielsson, J. Engquist, F. Kristiansson and P. Rajan for dis- 
cussions. We also thank E. Witten for stimulating correspondence. 


\section{References}

[1] B. Sundborg, Stringy gravity, interacting tensionless strings and massless higher spins, hep-th/0103247.

[2] P. Haggi-Mani and B. Sundborg, Free large N supersymmetric Yang-Mills theory as a string theory, JHEP 0004 (2000) 031, hep-th/0002189.

[3] E. Sezgin and P. Sundell, Doubletons and 5D higher spin gauge theory, hep-th/0105001.

[4] E. Sezgin and P. Sundell, Towards massless higher spin extension of $D=5, N=8$ gauged supergravity, JHEP 0109 (2001) 025, hep-th/0107186.

[5] M.A. Vasiliev, Conformal higher spin symmetries of $4 d$ massless supermultiplets and $\operatorname{osp}(L, 2 M)$ invariant equations in generalized (super) space, hep-th/0106149.

[6] M.A. Vasiliev, Cubic interactions of bosonic higher spin gauge fields in $A d S_{5}$, hep-th/0106200.

[7] E. Witten, talk given at J.H. Schwarz' 60th Birthday Conference, Cal Tech, Nov 2-3, 2001.

[8] A. Mikhailov, Notes on higher spin symmetries, hep-th/0201019.

[9] E. Sezgin and P. Sundell, 7D bosonic higher spin theory: symmetry algebra and linearized constraints, hep-th/0112100.

[10] E. Sezgin and P. Sundell, Massless higher spins and holography, hep-th/0205131.

[11] S. S. Gubser, I. R. Klebanov and A. M. Polyakov, A semi-classical limit of the gauge/string correspondence, hep-th/0204051.

[12] M.A. Vasiliev, Consistent equations for interacting gauge fields of all spins in $3+1$ dimensions, Phys. Lett. B243(1990) 378.

[13] M.A. Vasiliev, Higher spin gauge theories: star-product and AdS space, hep-th/9910096.

[14] U. Danielsson, F. Kristiansson, P. Rajan, E. Segin and P. Sundell, work in progress.

[15] E. Sezgin and P. Sundell, Higher spin N=8 supergravity, JHEP 9811 (1998) 016, hep-th/9805125.

[16] E. Sezgin and P. Sundell, Higher spin $N=8$ supergravity in $A d S_{4}$, hep-th/9903020. 
[17] M. Flato and C. Fronsdal, One massless particle equals two Dirac singletons, Lett. Math. Phys. 2 (1978) 421.

[18] M.A. Vasiliev, Dynamics of massless higher spins in the second order in curvatures, Phys. Lett. B238 (1990) 305.

[19] E. Sezgin and P. Sundell, On curvature expansion of higher spin gauge theory, hep-th/0012168.

[20] M.A. Vasiliev, Higher spin gauge theories in four, three and two dimensions, Int. J. Mod. Phys. D5 (1996) 763, hep-th/9611024.

[21] S.E. Konstein and M.A. Vasiliev, Extended higher-spin superalgebras and their massless representations, Nucl. Phys. B331 (1990) 475.

[22] S.E. Konstein, M.A. Vasiliev and V.N. Zaikin, Conformal higher spin currents in any dimension and AdS/CFT correspondence, JHEP 0012 (2000) 018, hep-th/0010239.

[23] J. Engquist, E. Sezgin and P. Sundell, On $\mathcal{N}=1,2,4$ higher spin gauge theories in four dimensions, hep-th/0207101.

[24] C. Fronsdal, Dirac supermultiplet, Phys. Rev. D26 (1982) 1988.

[25] H. Nicolai, E. Sezgin and Y. Tanii, Conformally invariant supersymmetric field theories on $S^{p} \times S^{1}$ and super p-branes, Nucl. Phys. B305 (1988) 483 .

[26] E. Sokatchev, Off-shell singletons, Class. Quant. Grav. 6 (1989) 93.

[27] E. Sezgin and Y. Tanii, Superconformal invariant sigma models in higher than two-dimensions, Nucl. Phys. B443 (1995) 70, hep-th/9412163.

[28] S. Prokushkin and M.A. Vasiliev, Higher-spin gauge interactions for massive matter fields in 3D AdS space-time, Nucl. Phys. B545 (1999) 385, hep-th/9806236.

[29] M.P. Blencowe, A consistent interacting massless higher spin field theory in $D=2+1$, Class. Quant. Grav. 6 (1989) 443. 